\title{
NASH INEQUALITIES FOR COMPACT MANIFOLDS WITH BOUNDARY
}

\author{
HiRONORI KUMURA*
}

\begin{abstract}
In this paper, we shall prove the Nash inequality for a compact manifold with boundary with respect to a weighted measure, using covering arguments of Jerison [10] and Oden-Sung-Wang [13]. We shall also state some results which are easily obtained from that inequality.
\end{abstract}

\section{Introduction}

The Nash inequality is equivalent not only to the Sobolev inequality but also to the diagonal upper bound of the heat kernel. Therefore, it is an important factor for the study of solutions of parabolic equations ([7]). In this paper, we shall first prove the Nash inequality for metric balls under the assumptions of the volume doubling property and local Poincaré inequality, by using covering arguments of Jerison [10]. We shall also point out that the Nash inequality, conversely, implies a lower bound of the volume of balls, and hence, a family of Nash inequalities is equivalent to the parabolic Harnack inequality. Using covering arguments of K. Oden, C. J. Sung and J. Wang [13], we also derive the Nash inequality for a compact manifold with boundary with respect to a weighted measure $w v_{M}$, where $w$ is a positive function on Int $M:=M-\partial M$.

We shall make the above statements mathematically precise. Let $M$ be a compact, connected Riemannian manifold with boundary $\partial M$. We denote by $v_{M}$ the Riemannian measure of $M$. In order to emphasize that $M$ contains the boundary $\partial M$, we shall often write $\bar{M}$ in place of $M$. K. Oden, C. J. Sung and J. Wang proved the Poincare inequality of $M$ with respect to a weighted measure;

Theorem 1.1 (K. Oden, C. J. Sung and J. Wang [13]). Let $w$ be a given function on $M$ with $w>0$ on Int $M:=M-\partial M$. We assume

1. $M$ satisfies the volume doubling property, i.e., $\exists c_{1}>0$ s.t. for any ball $B(x, 2 r)$ with $x \in \bar{M}$ we have $|B(x, 2 r)| /|B(x, r)| \leq c_{1}$, where $c_{1}$ is a constant in-

* Partly supported by the Grant-in-Aid for Scientific Research, No. 11740038, The Ministry of Education, Science and Culture, Japan

Received September 4, 2000; revised July 26, 2001. 
dependent of $x$ and $r$, and for $A \subset M,|A|$ stands for the volume of $A$, i.e., $|A|=\int_{A} d v_{M}$.

2. $M$ satisfies the interior rolling $R$-ball condition, i.e., $\exists R>0$ s.t. for all $x \in \partial M, \exists B(p, R) \subset M$ s.t. $B(p, R) \cap \partial M=\{x\}$.

3. $M$ satisfies the weak Poincaré inequality on balls, i.e., for any ball $B(x, 2 r) \subset M, B(x, 2 r) \cap \partial M=\emptyset$, we have

$$
\inf _{k \in \boldsymbol{R}} \int_{B(x, r)}|f-k|^{2} d v_{M} \leq c_{2} r^{2} \int_{B(x, 2 r)}|\nabla f|^{2} d v_{M}
$$

for all $f \in C^{1}(\bar{M})$, where $c_{2}$ is a constant independent of $x, r$ and $f$.

4. $\exists c_{3}>1$ s.t. $w(x) \leq c_{3} w(y)$ for all $x, y \in M$ with $0<d(x, \partial M) \leq 2 d(y, \partial M)$.

Then we have the following Poincaré inequality on $M$ :

$$
\inf _{f \in C^{1}(\bar{M})} \frac{\int_{M}|\nabla f|^{2} w d v_{M}}{\inf _{k \in \boldsymbol{R}} \int_{M}|f-k|^{2} w d v_{M}} \geq c\left(c_{1}, c_{2}, c_{3}, R, \eta, D\right),
$$

where $\eta$ is the first nonzero Neumann eigenvalue of $M_{R / 2}=\{x \in M \mid d(x, \partial M) \geq$ $R / 2\}$ and $D=\operatorname{diam}(M)$, the diameter of $M$.

We shall consider

5. $\exists c_{6}>0$ and $\exists \varphi>0$ s.t. $c_{6} d(x, \partial M)^{2 \varphi} \leq w(x)$ for all $x \in M-M_{R}$, where $M_{R}=\{x \in M \mid d(x, \partial M) \geq R\}$.

In this paper, we shall add this assumption 5 to the ones of Theorem 1.1, and prove the Nash inequality,

$$
\left(\int_{M}\left|f-f_{M}^{\prime}\right|^{2} w d v_{M}\right)^{1+(2 / v)} \leq c \cdot\left(\int_{M}|\nabla f|^{2} w d v_{M}\right)\left(\int_{M}\left|f-f_{M}^{\prime}\right| w d v_{M}\right)^{4 / v},
$$

$\forall v$ satisfying $v>4 \varphi$ and $v \geq 2 v_{0}:=2\left(\log c_{1}\right) / \log 2, \quad \forall f \in C^{\infty}(M)$,

where

$$
\begin{gathered}
f_{M}^{\prime}=\int_{M} f w d v_{M} / \int_{M} w d v_{M}, \\
c=c\left(c_{1}, c_{2}, c_{3}, c_{6}, v, \varphi, R, D,|M|\right)+c\left(c_{3}, c_{6}, v, \varphi, R, D\right) \cdot N,
\end{gathered}
$$

$|M|$ stands for the volume of $M$, i.e., $|M|=\int_{M} d v_{M}$ and $N$ is the "Nash constant" of $M_{R / 2}$ (see equation (7) in Theorem 4.1).

We should note that, actually, the assumption 4 implies the assumption 5 with $c_{6}=R^{-2 \varphi} \inf _{d(x, \partial M)=R / 2} w(x)$ and $2 \varphi=\left(\log c_{3}\right) / \log 2$, and hence the assumption 5 is not required for a Nash inequality to hold (cf. Remark 4.1). Nevertheless we assume the property 5 because it is important to choose $v$ as small as possible.

Let $u_{1}$ be the normalized first Dirichlet eigenfunction for the Laplacian on $M$. K. Oden, C. J. Chen and J. Wang set $w=\left(u_{1}\right)^{2}$ and estimated the constant $c\left(c_{1}, c_{2}, c_{3}, R, \eta, D\right)$ in Theorem 1.1 from below by the geometric constants of $M$. 
Similarly, the above constant $c$ can be estimated from above by the geometric constants of $M$ (see Proposition 5.1).

In section 3, we shall also point out that the Nash inequality conversely means the lower bound of the measure in the following way: Let $M$ be the closure of a relatively compact domain in a complete Riemannian manifold $N, w$ be a function such that $w \in C^{\infty}(M)$ and $0<w$ on Int $M=M-\partial M$. ( $w$ may possibly takes zero-value at a point of the boundary $\partial M$ ). We suppose that the following Nash inequality holds:

$$
\begin{aligned}
& \left(\int_{M}\left|f-f_{M}^{\prime}\right|^{2} d \mu^{w}\right)^{1+(2 / v)} \\
& \quad \leq \hat{c} \cdot(\operatorname{diam} M)^{2} \cdot \mu^{w}(M)^{-2 / v}\left(\int_{M}|\nabla f|^{2} d \mu^{w}\right)\left(\int_{M}\left|f-f_{M}^{\prime}\right| d \mu^{w}\right)^{4 / v},
\end{aligned}
$$

for all $f \in C^{\infty}(M)$, where $f_{M}^{\prime}=\int_{M} f d \mu^{w} / \mu^{w}(M)$. Then, for each $0<r<\operatorname{diam} M$ and $x_{0} \in M$, we have the lower bound of the measure

$$
\frac{\mu^{w}\left(B\left(x_{0}, r\right) \cap M\right)}{\mu^{w}(M)} \geq \frac{c(v)}{1+\hat{c}^{v / 2}}\left(\frac{r}{\operatorname{diam} M}\right)^{v} .
$$

From this lower bound estimate of the measure, we can easily see that the parabolic Harnack inequality (i.e., Theorem 3.3,2) is equivalent to a family of Nash inequalities, i.e., there exist constants $\hat{c}>0, r_{0}>0$ and $v>0$ such that

$$
\begin{gathered}
\left(\int_{B(x, r)}\left|f-f_{B(x, r)}^{\prime}\right|^{2} d \mu^{w}\right)^{1+(2 / v)} \\
\leq \hat{c} \cdot r^{2} \cdot \mu^{w}(B(x, r))^{-2 / v}\left(\int_{B(x, r)}|\nabla f|^{2} d \mu^{w}\right)\left(\int_{B(x, r)}\left|f-f_{B(x, r)}^{\prime}\right| d \mu^{w}\right)^{4 / v}, \\
\forall f \in C^{\infty}(\overline{B(x, r)}), \quad \forall x \in M, 0<\forall r<r_{0} .
\end{gathered}
$$

The author would like to express his gratitude to Professor Atsushi Kasue for his directing the author's attention to the paper [13] of K. Oden, C. J. Sung and J. Wang.

\section{Abstract results}

In this section, we shall state the equivalence between the diagonal heat kernel upper bound, Nash inequality, and Sobolev inequality. We shall also point out that the diagonal heat kernel upper bound implies a lower bound of the eigenvalue of the associated operator.

Let $M$ be a compact, connected Riemannian manifold with boundary $\partial M$ and $w \in C^{0}(\bar{M})$ be a function which is positive on Int $M:=M-\partial M$. We define a weighted measure $\mu^{w}$, by $\mu^{w}(E)=\int_{E} w d v_{M}$ for $E \subset M$. We denote by $H^{1}\left(M, \mu^{w}\right)$ 
a Hilbert space obtained by the completion of the space $C^{0}(M) \cap C^{\infty}($ Int $M)$ with respect to the norm

$$
\|f\|_{H^{1}\left(M, \mu^{w}\right)}^{2}=\int_{M}\left(|\nabla f|^{2}+f^{2}\right) d \mu^{w} .
$$

On the Hilbert space $L^{2}\left(M, \mu^{w}\right)$, we shall consider a closed form

$$
\mathscr{E}(f, h)=\int_{M}\langle\nabla f, \nabla h\rangle d \mu^{w} ; \quad f, h \in \operatorname{Dom}(\mathscr{E})=H^{1}\left(M, \mu^{w}\right)
$$

and denote by $A$ the nonpositive self-adjoint operator associated with this closed form $\mathscr{E}$. Let $p_{t}(x, y)$ be the heat kernel of the operator $A$. Slight modifications of the arguments in [2] and [16] enable us to show the following

Theorem 2.1. Let $M, w, A$, and $p_{t}$ be as above. We assume $\int_{M} w d v_{M}=1$ for simplicity. Let $v>0$ be given. Then the following inequalities (1) and (3) are equivalent, and when $v>2$, they are also equivalent with (2) and (4):

(1) $p_{t}(x, x) \leq 1+a t^{-v / 2} \quad(\forall t>0, \forall x \in M)$.

(2) $\|f\|_{2 v /(v-2)}^{2} \leq b \cdot \mathscr{E}(f, f) \quad \forall f \in C^{0}(M) \cap C^{\infty}(\operatorname{Int}(M))$ with $\int_{M} f w d v_{M}=0$.

(3) $\|f\|_{2}^{2+(4 / v)} \leq c \cdot \mathscr{E}(f, f)\|f\|_{1}^{4 / v} \quad \forall f \in C^{0}(M) \cap C^{\infty}(\operatorname{Int}(M)) w i t h \int_{M} f w d v_{M}=0$.

(4) $\inf _{\alpha \in \boldsymbol{R}}\|f-\alpha\|_{2 v /(v-2)}^{2} \leq d \cdot \mathscr{E}(f, f) \quad \forall f \in C^{0}(M) \cap C^{\infty}(\operatorname{Int}(M))$.

Here, we write $\|f\|_{p}=\left(\int_{M}|f|^{p} w d v_{M}\right)^{1 / p}$. (3) implies (1) with $a=c_{1} v^{v / 2} c^{v / 2}$, (1) implies (3) with $c=c_{2} a^{2 / v}$, (1) implies (2) with $b=c_{3} v^{2}(v-2)^{-2} a^{2 / v}$, (2) implies (3) with $c=b$, (2) implies (4) with $d=b$, and (4) implies (2) with $b=4 d$, where $c_{1}, c_{2}, c_{3}$ are some numerical constants.

COROLlary 2.1. The Nash inequality (3) in Theorem 2.1 implies

$$
\alpha^{-2 / v} v^{-1} c^{-1} i^{2 / v} \leq l_{i}, \quad\left|\varphi_{i}\right|_{\infty}^{2} \leq e \alpha\left(v c l_{i}\right)^{v / 2} \quad(i=1,2, \ldots),
$$

where $0=l_{0}<l_{1} \leq l_{2} \leq \cdots$ are the eigenvalues of $-A$ and $\left\{\varphi_{i}\right\}$ is a complete orthonormal system of $L^{2}\left(M, \mu^{w}\right)$ consisting of eigenfunctions with $\varphi_{i}$ having eigenvalue $l_{i}$.

Proof. Theorem 2.1 implies $p_{t}(x, x) \leq 1+a t^{-v / 2}$ for all $t>0$ and $x \in M$, where $a=\alpha(v c)^{v / 2}$ and $\alpha$ is a numerical constant. Therefore for each $\lambda>0$ and for all $x \in M$,

$$
\begin{aligned}
\sum_{0<l_{i} \leq \lambda}\left(\varphi_{i}\right)^{2}(x) & \leq e \sum_{0<l_{i} \leq \lambda} e^{-l_{i} / \lambda}\left(\varphi_{i}\right)^{2}(x) \\
& \leq e\left\{p_{1 / \lambda}(x, x)-1\right\} \leq e a \lambda^{v / 2} .
\end{aligned}
$$

Integrating the both sides of this inequality, we get

$$
\sharp\left\{l_{i} \mid 0<l_{i} \leq \lambda\right\} \leq e a \lambda^{v / 2} \quad(\lambda>0) .
$$

Thus, Corollary 2.1 follows. 


\section{Nash inequality and volume comparison}

In this section, we shall prove the following three assertions:

1. The Nash inequality holds under the assumptions of the volume doubling property and weak Poincaré inequality.

2. The Nash inequality implies a volume comparison.

3. The local parabolic Harnack inequality is equivalent to the family of Nash inequalities.

Let $M$ be a complete Riemannian manifold and $\mu$ be a positive Borel measure on $M$. In this section, we shall write $V(x, r)=\mu(B(x, r)),|A|=\mu(A)$ and $f_{A}=\mu(A)^{-1} \int_{A} f d \mu$, for a bounded measurable subset $A \subseteq M$ and a function $f \in C^{0}(\bar{A})$.

Lemma 3.1. Let $M$ and $\mu$ be as above. We assume the following condition $(D)$ and $(P)$ :

(D) $V(x, 2 r) \leq c_{1} V(x, r)$ for all $x \in M$ and $0<r \leq r_{0}$, where $c_{1}$ is a constant independent of $x$ and $r$;

(P) $\int_{B(x, r)}\left|f-f_{B(x, r)}\right|^{2} d \mu \leq c_{2} r^{2} \int_{B(x, 2 r)}|\nabla f|^{2} d \mu$ for all $x \in M, \quad 0<r \leq r_{0}$ and $f \in C^{\infty}(M)$, where $c_{2}$ is a constant independent of $x, r$ and $f$.

Then, for each $v \geq v_{0}:=\left(\log c_{1}\right) / \log 2$, we have

$$
\|f\|_{L^{2}(B(x, r), \mu)} \leq c_{4} s\|\nabla f\|_{L^{2}(B(x, 8 r), \mu)}+c_{5} V(x, r)^{-1 / 2}(r / s)^{v / 2}\|f\|_{L^{1}(B(x, r), \mu)},
$$

for all $r \in\left(0, r_{0}\right), s>0$, and $f \in C^{\infty}(\overline{B(x, 8 r)})$ with $\int_{B(x, r)} f d \mu=0$, where $c_{4}$ and $c_{5}$ are constants depending only on $c_{1}$ and $c_{2}$.

Proof. From the proof of Theorem 2.1 in Saloff-Coste [14], we see that there exist constants $c_{6}$ and $c_{7}$ depending only on $c_{1}$ and $c_{2}$ such that

$$
\int_{B(x, r)} f^{2} d \mu \leq c_{6} s^{2} \int_{B(x, 8 r)}|\nabla f|^{2} d \mu+c_{7} V(x, r)^{-1}\left(\frac{r}{s}\right)^{v}\left(\int_{B(x, r)}|f| d \mu\right)^{2}
$$

for all $f \in C^{\infty}(\overline{B(x, 8 r)}), 0<r<r_{0}, 0<s \leq r / 4$, and $v \geq v_{0}$. Since $\int_{B(x, r)} f d \mu=0$, the assumption $(P)$ implies

$$
\int_{B(x, r)} f^{2} d \mu \leq c_{2} r^{2} \int_{B(x, 2 r)}|\nabla f|^{2} d \mu \leq c_{6} S^{2} \int_{B(x, 8 r)}|\nabla f|^{2} d \mu
$$

for $s \geq \sqrt{c_{2} / c_{6}} r$. Hence, when we replace $c_{6}$ with $\max \left\{c_{6}, 16 c_{2}\right\}$ if necessary, the inequality (1) holds for all $s>0$. This proves Lemma 3.1.

We shall set $E:=B\left(\xi_{1}, r_{1}\right) \subset M$ and consider the following conditions $(V D)_{1}$ and $(V D)_{2}$ :

$(V D)_{1} M$ satisfies the volume doubling condition, i.e., $V(x, 2 r) \leq c_{1} V(x, r)$ for $x \in M, 0<r \leq r_{0}$

$(V D)_{2} \mu(B(x, 2 r) \cap E) \leq c_{1} \mu(B(x, r) \cap E)$ for $x \in \bar{E}, 0<r \leq r_{0}$.

In order to show the first assertion 1, we shall use the following covering lemma due to Jerison [10]: 
Lemma 3.2 (Whitney decomposition [10]). We set $E:=B\left(\xi_{1}, r_{1}\right) \subset M$ and assume $(V D)_{1}$ or $(V D)_{2}$. Then there exist a pairwise disjoint family $\mathscr{F}=$ $\left\{B_{i}=B\left(x_{i}, r_{i}\right) \mid i \in I\right\}$ of metric balls of $E$ and a constant $c\left(c_{1}, r_{1} / r_{0}\right)$ such that the following assertions hold:

(1) $E=\bigcup_{i \in I} 2 B_{i}$;

(2) $10^{2} \rho\left(B_{i}\right) \leq \operatorname{dist}\left(B_{i}, \partial E\right) \leq 10^{3} \rho\left(B_{i}\right) \quad(\forall i \in I)$;

(3) $\sharp\left\{i \in I \mid \eta \in 32 B_{i}\right\} \leq c\left(c_{1}, r_{1} / r_{0}\right) \quad(\forall \eta \in E)$,

where $c\left(c_{1}, r_{1} / r_{0}\right)$ is a constant depending only on the constants $c_{1}$ and $\max \left\{1, r_{1} / r_{0}\right\}$, for $B=B(x, r)$ and $a>0$, we write $a B:=B(x$, ar $)$, and $\rho(B)$ stands for the radius of $B$.

For $B \in \mathscr{F}$, let $\gamma_{B}$ be a geodesic segment from the center of $B$ to the center $\xi_{1}$ of $E$. (This path may not be unique, but will be fixed throughout our arguments). Denote $\mathscr{F}(B)=\left\{A \in \mathscr{F} \mid 2 A \cap \gamma_{B} \neq \emptyset\right\}$ and $A(\mathscr{F})=\{B \in \mathscr{F} \mid A \in \mathscr{F}(B)\}$ for $A \in \mathscr{F}$. Then the following holds:

(4) $\sum_{B \in A(\mathscr{F})} \sharp \mathscr{F}(B) \frac{|B|}{|A|} \leq c\left(c_{1}\right) \cdot \log \left(\frac{r_{1}}{\rho(A)}\right)$.

Lemma 3.3. Let $E=B\left(\xi_{1}, r_{1}\right)$ and $M$ be as in Lemma 3.2. We assume one of the two conditions $(V D)_{1}$ and $(V D)_{2}$. Moreover, we suppose that

$$
\begin{aligned}
& \int_{B(x, r)}\left|f-f_{B(x, r)}\right|^{2} d \mu \\
& \quad \leq c_{4} s^{2} \int_{B(x, 8 r)}|\nabla f|^{2} d \mu+c_{5} V(x, r)^{-1}\left(\frac{r}{S}\right)^{v}\left(\int_{B(x, r)}\left|f-f_{B(x, r)}\right| d \mu\right)^{2},
\end{aligned}
$$

for all $B(x, 8 r) \subset E, s>0, f \in C^{\infty}(\overline{B(x, 8 r)})$ and $v \geq v_{0}:=\left(\log c_{1}\right) / \log 2$. Then, if $2 r_{1} \leq r_{0}$, or if

$$
|E|=V\left(\xi_{1}, r_{1}\right) \leq c_{3}|A|\left(\frac{2 r_{1}}{\rho(A)}\right)^{v_{0}} \quad \text { for all ball } A \subset E,
$$

then we have the Nash inequality:

$$
\left\|f-f_{E}\right\|_{L^{2}(E)}^{2+\left(4 / v^{\prime}\right)} \leq c\left(c_{1}, c_{4}, c_{5}, r_{1} / r_{0}, v^{\prime}\right) \cdot\left(r_{1}\right)^{2}\left(c_{3}\right)^{2 / v^{\prime}}|E|^{-2 / v^{\prime}}\|\nabla f\|_{L^{2}(E)}^{2}\left\|f-f_{E}\right\|_{L^{1}(E)}^{4 / v^{\prime}},
$$

for all $f \in C^{\infty}(\bar{E})$, where $v^{\prime}=2 v$.

Remark 3.1. We note that the inequality (2) follows from the fact that $2 r_{1} \leq r_{0}$. Indeed, we first observe that the property $(V D)_{1}$ or $(V D)_{2}$ implies that $V(x, r) \leq c_{1} V(x, s)(r / s)^{v_{0}}$ for $0<s<r \leq r_{0}$. We denote by $o_{A}$ the center of a ball $A$. Since $A \subset E$ implies $E=B\left(\xi_{1}, r_{1}\right) \subset B\left(o_{A}, 2 r_{1}\right)$, we have

$$
V\left(\xi_{1}, r_{1}\right) \leq V\left(o_{A}, 2 r_{1}\right) \leq c_{1} V\left(o_{A}, \rho(A)\right)\left(2 r_{1} / \rho(A)\right)^{v_{0}},
$$

and hence, the inequality (2) with $c_{3}=c_{1}$ holds. 
Proof. Let $\mathscr{F}$ be a Whitney decomposition as in Lemma 3.2 and $f \in C^{\infty}(\bar{E})$. We choose $B_{0} \in \mathscr{F}$ such that $2 B_{0} \ni \xi_{1}$. For $B \in \mathscr{F}$, there exist $A_{1}, \ldots, A_{l} \in \mathscr{F}(B)$ such that

$$
A_{1}=B, \quad A_{l}=B_{0}, \quad 2 A_{i} \cap 2 A_{i+1} \neq \emptyset \quad(i=1,2, \ldots, l-1) .
$$

By virture of $(V D),(N)$, and Lemma $3.2(2)$, we have

$$
\int_{4 A_{k}}\left|f-f_{4 A_{k}}\right|^{2} d \mu \leq I_{A_{k}}(s) \quad(\forall s>0),
$$

where we set

$$
I_{A_{k}}(s):=c_{4} s^{2} \int_{32 A_{k}}|\nabla f|^{2} d \mu+c_{6}\left|A_{k}\right|^{-1}\left(\frac{\rho\left(A_{k}\right)}{s}\right)^{v}\left(\int_{4 A_{k}}\left|f-f_{4 A_{k}}\right| d \mu\right)^{2},
$$

and $c_{6}=c\left(c_{1}, c_{5}\right)$. Hence

$$
\begin{aligned}
\mid f_{4 A_{k}} & -\left.f_{4 A_{k+1}}\right|^{2}\left|4 A_{k} \cap 4 A_{k+1}\right| \\
& =\int_{4 A_{k} \cap 4 A_{k+1}}\left|f_{4 A_{k}}-f_{4 A_{k+1}}\right|^{2} d \mu \\
& \leq 2 \int_{4 A_{k} \cap 4 A_{k+1}}\left|f-f_{4 A_{k}}\right|^{2}+\left|f-f_{4 A_{k+1}}\right|^{2} d \mu \leq 2 I_{k}\left(s_{k}\right)+2 I_{k+1}\left(s_{k+1}\right)
\end{aligned}
$$

for all $s_{k}>0$ and $s_{k+1}>0$, where $I_{k}:=I_{A_{k}}$.

Since $2 A_{k} \cap 2 A_{k+1} \neq \emptyset$, from Lemma 3.2 (2), we see that

$$
\frac{99}{10^{3}+3} \rho\left(A_{k+1}\right) \leq 99 \rho\left(A_{k}\right) \leq\left(10^{3}+3\right) \rho\left(A_{k+1}\right),
$$

and

$$
B\left(y, 2 \min \left\{\rho\left(A_{k}\right), \rho\left(A_{k+1}\right)\right\}\right) \subset 4 A_{k} \cap 4 A_{k+1},
$$

where $y \in 2 A_{k} \cap 2 A_{k+1}$. (In this proof of Lemma 3.3, integration is always with respect to the measure $\mu$, and hence, in the following, we shall often omit ' $d \mu$ ' for simplicity.) Therefore, it is easy to see that

$$
\begin{aligned}
& \int_{4 A_{k} \cup 4 A_{k+1}}\left|f-f_{4 A_{k}}\right|^{2} \\
& \quad \leq \int_{4 A_{k}}\left|f-f_{4 A_{k}}\right|^{2}+2 \int_{4 A_{k+1}}\left(\left|f-f_{4 A_{k+1}}\right|^{2}+\left|f_{4 A_{k+1}}-f_{4 A_{k}}\right|^{2}\right) \\
& \quad \leq I_{k}\left(s_{k}\right)+2 I_{k+1}\left(s_{k+1}\right)+4\left|4 A_{k+1}\right| \frac{I_{k}\left(s_{k}\right)+I_{k+1}\left(s_{k+1}\right)}{\left|4 A_{k} \cap 4 A_{k+1}\right|} \\
& \quad \leq c\left(c_{1}\right)\left\{I_{k}\left(s_{k}\right)+I_{k+1}\left(s_{k+1}\right)\right\} .
\end{aligned}
$$


Therefore, we get

$$
\int_{4 A_{k} \cup 4 A_{k+1}}\left|f_{4 A_{k}}-f_{4 A_{k+1}}\right|^{2} \leq c\left(c_{1}\right)\left\{I_{k}\left(s_{k}\right)+I_{k+1}\left(s_{k+1}\right)\right\} .
$$

Hence, by $(V D)$,

$$
\left|f_{4 A_{k}}-f_{4 A_{k+1}}\right|^{2} \leq \frac{c\left(c_{1}\right)}{\left|4 A_{k} \cup 4 A_{k+1}\right|}\left\{I_{k}\left(s_{k}\right)+I_{k+1}\left(s_{k+1}\right)\right\} \leq c\left(c_{1}\right)\left\{\frac{I_{k}\left(s_{k}\right)}{\left|A_{k}\right|}+\frac{I_{k}\left(s_{k+1}\right)}{\left|A_{k+1}\right|}\right\} .
$$

Therefore,

$$
\begin{aligned}
\int_{2 B}\left|f-f_{4 B_{0}}\right|^{2} & =\int_{2 B}\left|f-f_{4 B}+\sum_{k=1}^{l-1}\left(f_{4 A_{k}}-f_{4 A_{k+1}}\right)\right|^{2} \\
& \leq l \int_{2 B}\left(\left|f-f_{4 B}\right|^{2}+\sum_{k=1}^{l-1}\left|f_{4 A_{k}}-f_{4 A_{k+1}}\right|^{2}\right) \\
& \leq l\left\{I_{1}\left(s_{1}\right)+c\left(c_{1}\right) \sum_{k=1}^{l-1}\left(\frac{|B|}{\left|A_{k}\right|} I_{k}\left(s_{k}\right)+\frac{|B|}{\left|A_{k+1}\right|} I_{k+1}\left(s_{k+1}\right)\right)\right\} \\
& \leq c\left(c_{1}\right) \cdot l \sum_{k=1}^{l-1} \frac{|B|}{\left|A_{k}\right|} I_{k}\left(s_{k}\right) \\
& \leq c\left(c_{1}\right) \cdot \sharp \mathscr{F}(B) \sum_{A \in \mathscr{F}(B)} \frac{|B|}{|A|} I_{A}\left(s_{A}\right),
\end{aligned}
$$

for all $s_{A}>0$. Summing up all $B \in \mathscr{F}$, we get, by Lemma $3.2(1)$,

$$
\begin{aligned}
\int_{E}\left|f-f_{4 B_{0}}\right|^{2} & \leq \sum_{B \in \mathscr{F}} \int_{2 B}\left|f-f_{4 B_{0}}\right|^{2} \\
& \leq c\left(c_{1}\right) \sum_{B \in \mathscr{F}} \sharp \mathscr{F}(B) \sum_{A \in \mathscr{F}(B)} \frac{|B|}{|A|} I_{A}\left(s_{A}\right) \\
& =c\left(c_{1}\right) \sum_{A \in \mathscr{F}}\left(\sum_{B \in A(\mathscr{F})} \sharp \mathscr{F}(B) \frac{|B|}{|A|}\right) I_{A}\left(s_{A}\right) \\
& \leq c\left(c_{1}\right) \sum_{A \in \mathscr{F}} \log \left(\frac{r_{1}}{\rho(A)}\right) I_{A}\left(s_{A}\right)
\end{aligned}
$$

for all $s_{A}>0$, where we have used Lemma 3.2 (4). In this inequality, we shall set $s_{A}=\rho(A)^{1-\theta}|A|^{-\delta} s$ for $s>0, \theta \in \boldsymbol{R}$, and $\delta \in \boldsymbol{R}$. Then, we have 


$$
\begin{aligned}
\int_{E}\left|f-f_{4 B_{0}}\right|^{2} \leq & c\left(c_{1}, c_{4}\right) \sum_{A \in \mathscr{F}} \log \left(\frac{r_{1}}{\rho(A)}\right) \frac{\rho(A)^{2-2 \theta}}{|A|^{2 \delta}} s^{2} \int_{32 A}|\nabla f|^{2} \\
& +c\left(c_{1}, c_{5}\right) \sum_{A \in \mathscr{F}} \log \left(\frac{r_{1}}{\rho(A)}\right)|A|^{-1}\left(\rho(A)^{\theta}|A|^{\delta} s^{-1}\right)^{v}\left(\int_{4 A}\left|f-f_{4 A}\right|\right)^{2} \\
\leq & c\left(c_{1}, c_{4}\right) \cdot s^{2} \varepsilon_{1}^{-1}\left(r_{1}\right)^{\varepsilon_{1}} \sum_{A \in \mathscr{F}} \frac{\rho(A)^{2-2 \theta-\varepsilon_{1}}}{|A|^{2 \delta}} \int_{32 A}|\nabla f|^{2} \\
& +c\left(c_{1}, c_{5}\right) \cdot s^{-v}\left(\varepsilon_{2}\right)^{-1}\left(r_{1}\right)^{\varepsilon_{2}} \sum_{A \in \mathscr{F}} \frac{|A|^{\delta v-1}}{\rho(A)^{\varepsilon_{2}-\theta v}}\left(\int_{4 A}\left|f-f_{4 A}\right|\right)^{2}
\end{aligned}
$$

for all $\varepsilon_{1}, \varepsilon_{2}>0$, where we have used the fact that $(\log x) / x^{\varepsilon} \leq(e \varepsilon)^{-1}$ for all $x$, $\varepsilon>0$. Since

$$
\int_{4 A}\left|f_{E}-f_{4 A}\right|=|4 A|\left|f_{E}-f_{4 A}\right|=\left.|4 A||| 4 A\right|^{-1} \int_{4 A}\left(f-f_{E}\right)\left|\leq \int_{4 A}\right| f-f_{E} \mid,
$$

we have

$$
\int_{4 A}\left|f-f_{4 A}\right| \leq \int_{4 A}\left(\left|f-f_{E}\right|+\left|f_{E}-f_{4 A}\right|\right) \leq 2 \int_{4 A}\left|f-f_{E}\right| .
$$

Hence, noting Lemma 3.2 (3), we get

$$
\begin{aligned}
\sum_{A \in \mathscr{F}}|A|^{-1 / 2}\left(\int_{4 A}\left|f-f_{4 A}\right|\right)^{2} & \leq \sum_{A \in \mathscr{F}} 4|A|^{-1 / 2}\left(\int_{4 A}\left|f-f_{E}\right|\right)^{2} \\
& \leq c\left(c_{1}\right) \sum_{A \in \mathscr{F}}\left(\int_{4 A}\left|f-f_{E}\right|^{2}\right)^{1 / 2}\left(\int_{4 A}\left|f-f_{E}\right|\right) \\
& \leq c\left(c_{1}\right)\left(\int_{E}\left|f-f_{E}\right|^{2}\right)^{1 / 2} \sum_{A \in \mathscr{F}} \int_{4 A}\left|f-f_{E}\right| \\
& \leq c\left(c_{1}, r_{1} / r_{0}\right)\left(\int_{E}\left|f-f_{E}\right|^{2}\right)^{1 / 2} \int_{E}\left|f-f_{E}\right| \\
& \leq c\left(c_{1}, r_{1} / r_{0}\right)\left\{u \int_{E}\left|f-f_{E}\right|^{2}+u^{-1}\left(\int_{E}\left|f-f_{E}\right|\right)^{2}\right\}
\end{aligned}
$$

for all $u>0$. Now, we set

$$
\delta=1 /(2 v) ; \quad \theta=1 / 4 ; \quad \varepsilon_{1}=1 / 2 ; \quad \varepsilon_{2}=v / 4 .
$$

Then,

$$
\frac{|A|^{\delta v-1}}{\rho^{\varepsilon_{2}-\theta v}}=|A|^{-1 / 2}
$$


and from the assumption (2) and $v \geq v_{0}$,

$$
\frac{\rho(A)^{2-2 \theta-\varepsilon_{1}}}{|A|^{2 \delta}}=\frac{\rho(A)^{2-2 \theta-\varepsilon_{1}}}{|A|^{1 / v}} \leq\left(c_{3}\right)^{1 / v}|E|^{-1 / v} 2 r_{1} \rho(A)^{1-2 \theta-\varepsilon_{1}}=\left(c_{3}\right)^{1 / v}|E|^{-1 / v} 2 r_{1} .
$$

Therefore, we obtain, from Lemma 3.2 (3),

$$
\begin{aligned}
\int_{E}\left|f-f_{E}\right|^{2} \leq & \int_{E}\left|f-f_{4 B_{0}}\right|^{2} \\
\leq & c\left(c_{1}, c_{4}, r_{1} / r_{0}\right) \cdot s^{2}\left(\varepsilon_{1}\right)^{-1}\left(r_{1}\right)^{\varepsilon_{1}}\left(c_{3}\right)^{1 / v}|E|^{-1 / v} r_{1} \int_{E}|\nabla f|^{2} \\
& +c\left(c_{1}, c_{5}, r_{1} / r_{0}\right) \cdot s^{-v}\left(\varepsilon_{2}\right)^{-1}\left(r_{1}\right)^{\varepsilon_{2}}\left\{u \int_{E}\left|f-f_{E}\right|^{2}+u^{-1}\left(\int_{E}\left|f-f_{E}\right|\right)^{2}\right\}
\end{aligned}
$$

for all $s, u>0$, and hence

$$
\begin{aligned}
\left(1-c_{8} s^{-v}\left(\varepsilon_{2}\right)^{-1}\left(r_{1}\right)^{\varepsilon_{2}} u\right) \int_{E}\left|f-f_{E}\right|^{2} \leq & c_{7} s^{2}\left(\varepsilon_{1}\right)^{-1}\left(r_{1}\right)^{\varepsilon_{1}+1}\left(c_{3}\right)^{1 / v}|E|^{-1 / v} \int_{E}|\nabla f|^{2} \\
& +c_{8} s^{-v}\left(\varepsilon_{2}\right)^{-1}\left(r_{1}\right)^{\varepsilon_{2}} u^{-1}\left(\int_{E}\left|f-f_{E}\right|\right)^{2}
\end{aligned}
$$

for all $s, u>0$, where $c_{7}=c\left(c_{1}, c_{4}, r_{1} / r_{0}\right), \quad c_{8}=c\left(c_{1}, c_{5}, r_{1} / r_{0}\right)$. Hence, setting $u=2^{-1} c_{8}^{-1} s^{v} \varepsilon_{2}\left(r_{1}\right)^{-\varepsilon_{2}}$, we have, for all $s>0$,

$$
\begin{aligned}
\frac{1}{2} \int_{E}\left|f-f_{E}\right|^{2} \leq & c_{7} s^{2}\left(\varepsilon_{1}\right)^{-1}\left(r_{1}\right)^{\varepsilon_{1}+1}\left(c_{3}\right)^{1 / v}|E|^{-1 / v} \int_{E}|\nabla f|^{2} \\
& +2\left(c_{8}\right)^{2} s^{-2 v}\left(\varepsilon_{2}\right)^{-2}\left(r_{1}\right)^{2 \varepsilon_{2}}\left(\int_{E}\left|f-f_{E}\right|\right)^{2} .
\end{aligned}
$$

Here, we note that, in general, when $A$ and $B$ are positive constants, a function

$$
A s^{2}+B s^{-2 v}
$$

of $s>0$ takes the minimum value $A^{v /(v+1)} B^{1 /(v+1)}\left(v^{1 /(v+1)}+v^{-v /(v+1)}\right)$ when $s=(B v / A)^{1 /(2 v+2)}$. Hence, if we set

$$
A=2 c_{7}\left(\varepsilon_{1}\right)^{-1}\left(r_{1}\right)^{\varepsilon_{1}+1}\left(c_{3}\right)^{1 / v}|E|^{-1 / v} \int_{E}|\nabla f|^{2}
$$

and

$$
B=4\left(c_{8}\right)^{2}\left(\varepsilon_{2}\right)^{-2}\left(r_{1}\right)^{2 \varepsilon_{2}}\left(\int_{E}\left|f-f_{E}\right|\right)^{2}
$$

then we have

$$
\int_{E}\left|f-f_{E}\right|^{2} \leq c(v) A^{v /(v+1)} B^{1 /(v+1)} .
$$


Thus, recalling (3), we obtain

$$
\begin{aligned}
\left\|f-f_{E}\right\|_{L^{2}(E)}^{2+(2 / v)} & \leq c(v) A B^{1 / v} \\
& =c(v) \cdot c_{7}\left(c_{8}\right)^{2 / v}\left(c_{3}\right)^{1 / v}|E|^{-1 / v}\left(r_{1}\right)^{2}\|\nabla f\|_{L^{2}(E)}^{2}\left\|f-f_{E}\right\|_{L^{1}(E)}^{2 / v} \\
& =c\left(v, c_{1}, c_{4}, c_{5}, r_{1} / r_{0}\right)\left(c_{3}\right)^{1 / v}\left(r_{1}\right)^{2}|E|^{-1 / v}\|\nabla f\|_{L^{2}(E)}^{2}\left\|f-f_{E}\right\|_{L^{1}(E)}^{2 / v} .
\end{aligned}
$$

Hence, when we set $v^{\prime}=2 v$, we get

$$
\left\|f-f_{E}\right\|_{L^{2}(E)}^{2+\left(4 / v^{\prime}\right)} \leq c\left(v^{\prime}, c_{1}, c_{4}, c_{5}, r_{1} / r_{0}\right)\left(c_{3}\right)^{2 / v^{\prime}}\left(r_{1}\right)^{2}|E|^{-2 / v^{\prime}}\|\nabla f\|_{L^{2}(E)}^{2}\left\|f-f_{E}\right\|_{L^{1}(E)}^{4 / v^{\prime}} .
$$

This completes the proof of Lemma 3.3.

Putting Lemma 3.1 and 3.2 together, we have the following

THEOREM 3.1. Let $M$ be a complete Riemannian manifold, $\mu$ be a positive measure on $M$, and $E=B\left(\xi_{1}, r_{1}\right) \subset M$. We assume the following three conditions:

(1) (volume doubling condition)

$$
(\mathrm{VD})_{1} \quad V(x, 2 r) \leq c_{1} V(x, r) \quad \text { for } \forall x \in M, 0<\forall r \leq r_{0}
$$

or

$$
(\mathrm{VD})_{2} \quad \mu(B(x, 2 r) \cap E) \leq c_{1} \mu(B(x, r) \cap E) \quad \text { for } \forall x \in \bar{E}, \quad 0<\forall r \leq r_{0} ;
$$

(2) (weak Poincaré inequality)

$$
\int_{B(x, r)}\left|f-f_{B(x, r)}\right|^{2} d \mu \leq c_{2} r^{2} \int_{B(x, 2 r)}|\nabla f|^{2} d \mu
$$

for $\forall B(x, 2 r) \subset E, 0<\forall r \leq r_{0}, \forall f \in C^{\infty}(\bar{E})$;

(3) $2 r_{1} \leq r_{0}$ or

$$
|E|=V\left(\xi_{1}, r_{1}\right) \leq c_{3}|A|\left(\frac{2 r_{1}}{\rho(A)}\right)^{v_{0}} \quad \text { for all ball } A \subset E .
$$

Then we have the Nash inequality

$$
\left\|f-f_{E}\right\|_{L^{2}(E)}^{2+(4 / v)} \leq c\left(c_{1}, c_{2}, v, r_{0} / r_{1}\right)\left(r_{1}\right)^{2}\left(c_{3}\right)^{2 / v}|E|^{-2 / v}\|\nabla f\|_{L^{2}(E)}^{2}\left\|f-f_{E}\right\|_{L^{1}(E)}^{4 / v}
$$

for all $f \in C^{\infty}(\bar{E})$ and all $v \geq 2 v_{0}=2\left(\log c_{1}\right) / \log 2$. In this inequality, when $2 r_{1} \leq r_{0}, c\left(c_{1}, c_{2}, v, r_{0} / r_{1}\right)$ does not depend on $r_{0} / r_{1}$ and we can take $c_{3}=c_{1}$.

Remark 3.2. Theorem 2.1 implies that under the assumptions of Theorem 3.1, we also have the Sobolev inequality

$$
\inf _{\alpha \in \boldsymbol{R}}\|f-\alpha\|_{L^{2 v /(v-2)}(E)}^{2} \leq c\left(c_{1}, c_{2}, v, r_{0} / r_{1}\right)\left(r_{1}\right)^{2}\left(c_{3}\right)^{2 / v}|E|^{-2 / v}\|\nabla f\|_{L^{2}(E)}^{2} \quad\left(\forall f \in C^{\infty}(\bar{E})\right) .
$$

A Neumann eigenvalue estimate is also obtained by means of Corollary 2.1. 
Remark 3.3. After writing this paper, the author found that P. Hajłasz and P. Koskela [9] proved the Sobolev inequality in a more general situation than Theorem 3.1 by a different method. For this fact and other related results, see their paper.

The Nash inequality on $M$ implies a lower bound of the measure of the intersection of a ball and $M$. Thus, it restricts the boundary behavior of the measure from below;

THEOREM 3.2. Let $M$ be the closure of a relatively compact domain in a complete Riemannian manifold $N, w$ be a function such that $w \in C^{\infty}(M)$ and $0<w$ on Int $M=M-\partial M$ ( $w$ may possibly takes zero at a point of the boundary $\partial M)$. We suppose that there exists a constant $\hat{c}>0$ such that the following Nash inequality holds:

$$
\begin{aligned}
& \left(\int_{M}\left|f-f_{M}\right|^{2} d \mu^{w}\right)^{1+\left(2 / v^{\prime}\right)} \\
& \quad \leq \hat{c} \cdot(\operatorname{diam} M)^{2} \cdot \mu^{w}(M)^{-2 / v^{\prime}}\left(\int_{M}|\nabla f|^{2} d \mu^{w}\right)\left(\int_{M}\left|f-f_{M}\right| d \mu^{w}\right)^{4 / v^{\prime}},
\end{aligned}
$$

for all $f \in C^{\infty}(M)$, where $f_{M}=\int_{M} f d \mu^{w} / \mu^{w}(M)$. Then, for each $0<r<\operatorname{diam} M$ and $x_{0} \in M$, we have

$$
\frac{\mu^{w}\left(B_{M}\left(x_{0}, r\right)_{0}\right)}{\mu^{w}(M)} \geq \frac{c\left(v^{\prime}\right)}{1+\hat{c}^{v^{\prime} / 2}}\left(\frac{r}{\operatorname{diam} M}\right)^{v^{\prime}},
$$

where $c\left(v^{\prime}\right)$ is a constant depending only on $v^{\prime}$ and $B_{M}\left(x_{0}, r\right)_{0}$ stands for the connected component (containing $x_{0}$ ) of the intersection $B\left(x_{0}, r\right) \cap$ Int $M$ of the metric ball $B\left(x_{0}, r\right)$ of $N$ and Int $M$.

Proof. Let $p_{t}(x, y)$ be the heat kernel of the operator on $L^{2}\left(M, \mu^{w} / \mu^{w}(M)\right)$ associated with the closed form

$$
\mathscr{E}(f, h)=\int_{M}\langle\nabla f, \nabla h\rangle d \mu^{w} \quad\left(f, h \in \operatorname{Dom}(\mathscr{E})=H^{1}\left(M, \mu^{w}\right)\right) .
$$

Then, from Theorem 2.1, we have

$$
p_{t}(x, x) \leq 1+a t^{-v^{\prime} / 2} \quad(\forall t>0, \forall x \in M),
$$

where $a=\alpha\left(v^{\prime} \hat{c}\right)^{v^{\prime} / 2}(\operatorname{diam} M)^{v^{\prime}}$ and $\alpha$ is some numerical constant. Take a point $x_{0} \in M$ and $r>0$, and consider the form

$$
\mathscr{E}_{D}(f, h)=\int_{B_{M}\left(x_{0}, r\right)_{0}}\langle\nabla f, \nabla h\rangle d \mu^{w}
$$


for $f, h \in \operatorname{Dom}\left(\mathscr{E}_{D}\right):=\left\{f \in C^{\infty}\left(\overline{B_{M}\left(x_{0}, r\right)_{0}}\right) ;\left.f\right|_{\text {Int } M \cap \partial B_{M}\left(x_{0}, r\right)_{0}}=0\right\}$. Let $h_{t}(x, y)$ be the heat kernel on $L^{2}\left(B_{M}\left(x_{0}, r\right)_{0}, \mu^{w} / \mu^{w}\left(B_{M}\left(x_{0}, r\right)_{0}\right)\right)$ associated with the closure of $\mathscr{E}_{D}$. Then maximum principle implies that

$$
\begin{aligned}
h_{t}(x, y) & \leq \frac{\mu^{w}\left(B_{M}\left(x_{0}, r\right)_{0}\right)}{\mu^{w}(M)} p_{t}(x, y) \\
& \leq \frac{\mu^{w}\left(B_{M}\left(x_{0}, r\right)_{0}\right)}{\mu^{w}(M)}\left\{1+a t^{-v^{\prime} / 2}\right\}
\end{aligned}
$$

for $t>0$ and $x, y \in B_{M}\left(x_{0}, r\right)_{0}$. Hence, when we set $\tau_{0}=(\operatorname{diam} M)^{2}$ and $c_{4}=\left(\mu^{w}\left(B_{M}\left(x_{0}, r\right)_{0}\right) /\left(\mu^{w}(M)\right)\right)\left(a+\tau_{0}^{v^{\prime} / 2}\right), \quad$ we have, for $0<t \leq \tau_{0} \quad$ and $x, y \in B_{M}\left(x_{0}, r\right)_{0}$,

$$
h_{t}(x, y) \leq c_{4} t^{-v^{\prime} / 2} .
$$

Therefore, Theorem 2.2 in [14] implies that

$$
\begin{aligned}
& \left(\int_{B_{M}\left(x_{0}, r\right)_{0}}|f|^{2} \frac{d \mu^{w}}{\mu^{w}\left(B_{M}\left(x_{0}, r\right)_{0}\right)}\right)^{1+\left(2 / v^{\prime}\right)} \\
& \leq c_{6}\left(\int_{B_{M}\left(x_{0}, r\right)_{0}}|\nabla f|^{2} \frac{d \mu^{w}}{\mu^{w}\left(B_{M}\left(x_{0}, r\right)_{0}\right)}+\tau_{0}^{-1} \int_{B_{M}\left(x_{0}, r\right)_{0}}|f|^{2} \frac{d \mu^{w}}{\mu^{w}\left(B_{M}\left(x_{0}, r\right)_{0}\right)}\right) \\
& \quad \times\left(\int_{B_{M}\left(x_{0}, r\right)_{0}}|f| \frac{d \mu^{w}}{\mu^{w}\left(B_{M}\left(x_{0}, r\right)_{0}\right)}\right)^{4 / v^{\prime}}
\end{aligned}
$$

for all $f \in C^{\infty}\left(\overline{B_{M}\left(x_{0}, r\right)_{0}}\right)$ with $\left.f\right|_{\operatorname{Int} M \cap \partial B_{M}\left(x_{0}, r\right)_{0}}=0$, where $c_{6}=\alpha c_{4}^{2 / v^{\prime}}$ and $\alpha$ is some numerical constant. Hence, by the same arguments as in the proof of Lemma 2.5 in [11] (see also Akutagawa [1]), we can see that, for $0<s \leq \min \left\{\sqrt{\tau_{0}}, r\right\}$,

$$
\begin{aligned}
\frac{\mu^{w}\left(B_{M}\left(x_{0}, s\right)_{0}\right)}{\mu^{w}\left(B_{M}\left(x_{0}, r\right)_{0}\right)} & \geq c\left(v^{\prime}\right) c_{6}^{-v^{\prime} / 2} s^{v^{\prime}}=c\left(v^{\prime}\right) c_{4}^{-1} s^{v^{\prime}} \\
& =c\left(v^{\prime}\right) \frac{s^{v^{\prime}}}{a+\tau_{0}^{v^{\prime} / 2}} \frac{\mu^{w}(M)}{\mu^{w}\left(B_{M}\left(x_{0}, r\right)_{0}\right)} .
\end{aligned}
$$

Thus, setting $s=r \leq \sqrt{\tau_{0}}=\operatorname{diam} M$, we get

$$
\begin{aligned}
\frac{\mu^{w}\left(B_{M}\left(x_{0}, r\right)_{0}\right)}{\mu^{w}(M)} & \geq c\left(v^{\prime}\right) \frac{r^{v^{\prime}}}{c\left(v^{\prime}\right) \hat{c}^{v^{\prime} / 2}(\operatorname{diam} M)^{v^{\prime}}+c\left(v^{\prime}\right)(\operatorname{diam} M)^{v^{\prime}}} \\
& =\frac{c\left(v^{\prime}\right)}{1+\hat{c}^{v^{\prime} / 2}}\left(\frac{r}{\operatorname{diam} M}\right)^{v^{\prime}}
\end{aligned}
$$

for $0<r<\operatorname{diam} M$ and $x_{0} \in M$. 
Now, let $M$ be a complete Riemannian manifold and $w$ be a positive smooth function on $M$. Let positive constants $\hat{c}$ and $r_{0}$ be given, and let us assume that $M$ and $w$ satisfy a family of local Nash inequalities

$$
\begin{gathered}
\left\|f-f_{B\left(\xi_{1}, r_{1}\right)}\right\|_{L^{2}\left(B\left(\xi_{1}, r_{1}\right), \mu^{w}\right)}^{2+(4 / v)} \\
\leq \hat{c} \cdot\left(r_{1}\right)^{2} \cdot \mu^{w}\left(B\left(\xi_{1}, r_{1}\right)\right)^{-2 / v}\|\nabla f\|_{L^{2}\left(B\left(\xi_{1}, r_{1}\right), \mu^{w}\right)}^{2}\left\|f-f_{B\left(\xi_{1}, r_{1}\right)}\right\|_{L^{1}\left(B\left(\xi_{1}, r_{1}\right), \mu^{w}\right)}^{4 / v}, \\
\forall f \in C^{\infty}\left(\overline{B\left(\xi_{1}, r_{1}\right)}\right), \quad \forall \xi_{1} \in M, \quad 0<\forall r_{1}<r_{0} .
\end{gathered}
$$

Then, by Theorem 3.2, we have the volume comparison

$$
\frac{\mu^{w}\left(B\left(\xi_{1}, s\right)\right)}{\mu^{w}\left(B\left(\xi_{1}, r_{1}\right)\right)} \geq \frac{c(v)}{1+\hat{c}^{v / 2}}\left(\frac{s}{r_{1}}\right)^{v} \text { for } 0<\forall s<\forall r_{1} \leq r_{0}, \quad \forall \xi_{1} \in M .
$$

Moreover, we have local Poincaré inequalities

$$
\begin{gathered}
\left\|f-f_{B\left(\xi_{1}, r_{1}\right)}\right\|_{L^{2}\left(B\left(\xi_{1}, r_{1}\right), \mu^{w}\right)}^{2} \leq \hat{c} \cdot\left(r_{1}\right)^{2}\|\nabla f\|_{L^{2}\left(B\left(\xi_{1}, r_{1}\right), \mu^{w}\right)}^{2}, \\
\forall f \in C^{\infty}\left(\overline{B\left(\xi_{1}, r_{1}\right)}\right), \quad 0<\forall r_{1} \leq r_{0}, \quad \forall \xi_{1} \in M,
\end{gathered}
$$

because the Schwarz inequality implies that

$$
\mu^{w}\left(B\left(\xi_{1}, r_{1}\right)\right)^{-2 / v}\left\|f-f_{B\left(\xi_{1}, r_{1}\right)}\right\|_{L^{1}\left(B\left(\xi_{1}, r_{1}\right), \mu^{w}\right)}^{4 / v} \leq\left\|f-f_{B\left(\xi_{1}, r_{1}\right)}\right\|_{L^{2}\left(B\left(\xi_{1}, r_{1}\right), \mu^{w}\right)}^{4 / v} .
$$

Now, we recall the following theorem due to Salloff-Coste (see also A. A. Grigor'yan [8]):

THEOREM 3.3 (Salloff-Coste [14]). The following two properties 1 and 2 are equivalent.

1. The following properties (a) and (b) hold for some constants $r_{0}>0, c_{1}>0$ and $c_{2}>0$ :

(a) $\mu^{w}(B(x, 2 r)) \leq c_{1} \mu^{w}(B(x, r)), 0<\forall r<r_{0}, \forall x \in M$

(b) $\int_{B(x, r)}\left|f-f_{B(x, r)}\right|^{2} d \mu^{w} \leq c_{2} r^{2} \int_{B(x, 2 r)}|\nabla f|^{2} d \mu^{w}, \quad 0<\forall r<r_{0}, \quad \forall x \in M$, $\forall f \in C^{\infty}(M)$.

2. There exists $r_{1}>0$, and there exists a constant $c$ depending only on the parameters $0<\varepsilon<\eta<\delta<1$, such that, for any $x \in M$, any real $s$, and any $0<r<r_{1}$, any nonnegative solution $u$ of $\left(\partial_{t}+\mathscr{L}\right) u=0$ in $Q=\left(s-r^{2}, s\right) \times B(x, r)$ satisfies

$$
\sup _{Q_{-}}\{u\} \leq c \inf _{Q_{+}}\{u\}
$$

where $Q_{-}=\left[s-(\delta r)^{2}, s-(\eta r)^{2}\right] \times B(x, \delta r), Q_{+}=\left[s-(\varepsilon r)^{2}, s\right) \times B(x, \delta r)$ and $\mathscr{L} u=$ $-\Delta u-\langle\nabla \log w, \nabla u\rangle$.

As is shown above, a family of local Nash inequalities implies the properties (a) and (b) in Theorem 3.3, and hence, from Theorem 3.1 and 3.3 we see that a family of local Nash inequalities is also equivalent to local parabolic Harnack inequalities; 
COROLlary 3.1. The properties 1 and 2 in Theorem 3.3 are also equivalent to the following property 3:

3. There exist constants $\hat{c}>0, r_{0}>0$ and $v>0$ such that

$$
\begin{gathered}
\left\|f-f_{B(x, r)}\right\|_{L^{2}\left(B(x, r), \mu^{w}\right)}^{2+(4 / v)} \\
\leq \hat{c} \cdot r^{2} \cdot \mu^{w}(B(x, r))^{-2 / v}\|\nabla f\|_{L^{2}\left(B(x, r), \mu^{w}\right)}^{2}\left\|f-f_{B(x, r)}\right\|_{L^{1}\left(B(x, r), \mu^{w}\right)}^{4 / v}, \\
\forall f \in C^{\infty}(\overline{B(x, r)}), \quad \forall x \in M, 0<\forall r<r_{0} .
\end{gathered}
$$

\section{Nash inequality with respect to weighted measure}

This section is devoted to proving the following theorem:

THEOREM 4.1. Let $M$ be a compact Riemannian manifold with $C^{\infty}$ boundary $\partial M$ and $w$ be a positive $C^{\infty}$ function on Int $M:=M-\partial M$. $w$ possibly takes zero value at a point of $\partial M$. We assume the following properties $(D),(R),(P),\left(w_{1}\right)$ and $\left(w_{2}\right)$ :

(D) (volume doubling property) $|B(x, 2 r)| \leq c_{1}|B(x, r)|$ for all $x \in \bar{M}$ and $r>0$, where $|B(x, r)|=\int_{B(x, r)} d v_{M}$.

$(R) M$ satisfies the "interior rolling $R$-ball condition", that is, $\exists R>0 \quad \forall x \in$ $\partial M \exists B(p, R) \subset M$ s.t. $\overline{B(p, R)} \cap \partial M=\{x\}$.

$(P)$ (weak Poincaré inequality)

For all $B(x, 2 r) \subset M$ with $B(x, 2 r) \cap \partial M=\emptyset$, and all $f \in C^{\infty}(M)$,

$$
\inf _{\alpha \in \boldsymbol{R}} \int_{B(x, r)}|f-\alpha|^{2} d v_{M} \leq c_{2} r^{2} \int_{B(x, 2 r)}|\nabla f|^{2} d v_{M}
$$

where $c_{2}$ is a positive constant independent of $x, r$ and $f$.

$\left(w_{1}\right) \exists c_{3}>1$ s.t. $w(x) \leq c_{3} w(y)$ for all $x, y \in M$ with $0<d(x, \partial M) \leq$ $2 d(y, \partial M)$.

$\left(w_{2}\right) \exists c_{6}>0 \exists \varphi>0$ s.t. $c_{6} d(x, \partial M)^{2 \varphi} \leq w(x)$ for all $x \in M-M_{R}$, where $M_{R}=\{x \in M \mid d(x, \partial M) \geq R\}$.

Then we have the Nash inequality on M:

$$
\begin{aligned}
& \left(\int_{M}\left|f-f_{M}^{\prime}\right|^{2} w d v_{M}\right)^{1+(2 / v)} \\
& \quad \leq c \cdot\left(\int_{M}|\nabla f|^{2} w d v_{M}\right)\left(\int_{M}\left|f-f_{M}^{\prime}\right| w d v_{M}\right)^{4 / v}
\end{aligned}
$$

for all $v$ satisfying $v>4 \varphi$ and $v \geq 2 v_{0}$, and all $f \in C^{\infty}(\bar{M})$, where $f_{M}^{\prime}=$ $\int_{M} f d \mu^{w} / \mu^{w}(M)$

$$
\begin{aligned}
c= & c\left(c_{1}, c_{2}, c_{3}, c_{6}, v, \varphi\right) R^{(v-4 \varphi) / v}|M|^{-2 / v} D \\
& +c\left(c_{3}, D / R, v\right) c_{6}^{-2 / v} R^{-4 \varphi / v} c_{N}\left(M_{R / 2}\right)\left|M_{R / 2}\right|^{-2 / v} \operatorname{diam}\left(M_{R / 2}\right)^{2},
\end{aligned}
$$


$c_{N}\left(M_{R / 2}\right)$ is a constant satisfying

$$
\begin{gathered}
\left(\int_{M_{R / 2}}\left|f-f_{M_{R / 2}}\right|^{2} d v_{M}\right)^{1+(2 / v)} \leq N \int_{M_{R / 2}}|\nabla f|^{2} d v_{M} \cdot\left(\int_{M_{R / 2}}\left|f-f_{M_{R / 2}}\right| d v_{M}\right)^{4 / v}, \\
N:=c_{N}\left(M_{R / 2}\right) \cdot\left|M_{R / 2}\right|^{-2 / v} \cdot \operatorname{diam}\left(M_{R / 2}\right)^{2}, \quad \forall f \in C^{\infty}\left(\overline{M_{R / 2}}\right)
\end{gathered}
$$

$f_{M_{R / 2}}=\int_{M_{R / 2}} f d v_{M} /\left|M_{R / 2}\right|$, and $D$ stands for the diameter of $M$.

Remark 4.1. We should note that actually, the condition $\left(w_{2}\right)$ is not required for a Nash inequality to hold.

In fact, the condition $\left(w_{1}\right)$ implies, for example, the following inequality:

$$
w(x) \geq \frac{\inf \{w(x) \mid d(x, \partial M)=R / 2\}}{R^{2 \varphi_{1}}} d(x, \partial M)^{2 \varphi_{1}},
$$

where $2 \varphi_{1}=\left(\log c_{3}\right) / \log 2$. This can be seen as follows: Let us set $f(t)=$ $\inf \{w(x) \mid t \leq d(x, \partial M) \leq R\}$ for $0<t \leq R$. Then the function $f$ is nondecreasing and the condition $\left(w_{1}\right)$ implies that $f(2 t) / f(t) \leq c_{3}$ for $0<t \leq R / 2$. For $0<s<R$, if we take the integer $k$ such that $2^{k-1}<R / s \leq 2^{k}$, we see that

$$
f(R / 2) \leq f\left(2^{k-1} s\right) \leq\left(c_{3}\right)^{k-1} f(s)<(R / s)^{\left(\log c_{3}\right) / \log 2} f(s) .
$$

Hence, if we set $2 \varphi_{1}=\left(\log c_{3}\right) / \log 2$, then $\varphi_{1}>0$ and $f(s) \geq f(R / 2) R^{-2 \varphi_{1}} s^{2 \varphi_{1}}$. Therefore, if $d(x, \partial M)=s$, then $w(x) \geq f(s) \geq f(R / 2) R^{-2 \varphi_{1}} d(x, \partial M)^{2 \varphi_{1}}$.

Thus, when we set $c_{6}:=\inf \{w(x) \mid d(x, \partial M)=R / 2\} / R^{2 \varphi_{1}}$ and $\varphi:=\varphi_{1}$, the property $\left(w_{2}\right)$ above holds. In spite of this fact, we assume the condition $\left(w_{2}\right)$ because it is important to choose $v>0$ as small as possible. Indeed, in Proposition 5.1, we shall consider the weight function $w=\left(u_{1}\right)^{2}$, where $u_{1}$ is the normalized first Dirichlet eigenfunction of $M$, and set $\varphi=1$.

Remark 4.2. As is seen from the following proof of Theorem 4.1, in order to get the Nash inequality (6), it suffice to assume that the doubling property $(D)$ and weak Poincare inequality $(P)$ hold only on the neighborhood $M-M_{R}$ of the boundary $\partial M$.

COROllary 4.1. Let $M$ be a metric ball of radius $R$ in a complete Riemannian manifold and assume $(D),(P),\left(w_{1}\right)$, and $\left(w_{2}\right)$. Then the Nash inequality (6) with $R=D$ and $c_{N}\left(M_{R / 2}\right)=0$, holds.

Remark 4.3. As is seen from Theorem 2.1, the Nash inequality (6) is equivalent to the following Sobolev inequality:

$$
\begin{gathered}
\inf _{\alpha \in \boldsymbol{R}}\left(\int_{M}|f-\alpha|^{2 v /(v-2)} d \mu^{w}\right)^{(v-2) / v} \leq c \mu^{w}(M)^{-2 / v} \int_{M}|\nabla f|^{2} d \mu^{w}, \\
\forall v \text { satisfying } v>4 \varphi \text { and } v \geq 2 v_{0}, \quad \forall f \in C^{\infty}(\bar{M}) .
\end{gathered}
$$


The inequality (7) is also equivalent to the following:

$$
\begin{aligned}
& \inf _{\alpha \in \boldsymbol{R}}\left(\int_{M_{R / 2}}|f-\alpha|^{2 v /(v-2)} d v_{M}\right)^{(v-2) / v} \\
& \quad \leq c_{N}\left(M_{R / 2}\right) \cdot\left|M_{R / 2}\right|^{-2 / v} \cdot \operatorname{diam}\left(M_{R / 2}\right)^{2} \int_{M_{R / 2}}|\nabla f|^{2} d v_{M}, \quad \forall f \in C^{\infty}\left(\overline{M_{R / 2}}\right) .
\end{aligned}
$$

In order to prove Theorem 4.1, we shall use the following covering lemma due to Oden, Sung and Wang:

Lemma 4.1 (Whitney decomposition [13]). Let $M$ be a compact Riemannian manifold with boundary which satisfies the doubling property $(D)$. Then, there exists a pairwise disjoint family $\mathscr{F}=\left\{B_{i}=B\left(x_{i}, r_{i}\right) \mid i \in I\right\}$ of geodesic balls in Int $M$ satisfying the following:

(1) $\bigcup_{i \in I} 2 B_{i}=\operatorname{Int} M$;

(2) $\operatorname{dist}\left(B\left(x_{i}, r_{i}\right), \partial M\right)=10^{3} r_{i}$;

(3) There exists a constant $c\left(c_{1}\right)$ depending only on $c_{1}$ such that, for all $\eta \in \operatorname{Int} M, \sharp\left\{B_{i} \in \mathscr{F} \mid \eta \in 32 B_{i}\right\} \leq c\left(c_{1}\right)$.

Denote $M_{R}:=\{x \in M \mid d(x, \partial M) \geq R\}$ and $\mathscr{L}:=\left\{B_{i} \in \mathscr{F} \mid x_{i} \notin M_{R}\right\} . \quad$ For $B_{i}=B\left(x_{i}, r_{i}\right) \in \mathscr{L}$, the interior rolling $R$-ball condition implies that there exist $y_{i} \in \partial M$ and $B\left(q_{i}, R\right) \subset \operatorname{Int}(M)$ such that $d\left(x_{i}, y_{i}\right)=d\left(x_{i}, \partial M\right)$ and $\overline{B\left(q_{i}, R\right)} \cap \partial M=$ $\left\{y_{i}\right\}$. Let $\overline{q_{i} y_{i}}$ be the minimal geodesic segment from $q_{i} \in \partial M_{R}$ to $y_{i}$. Then $x_{i} \in \overline{q_{i} y_{i}}$. Denote by $l_{i}$ the segment $\overline{q_{i} x_{i}}$ of $\overline{q_{i} y_{i}}$. For $B_{i} \in \mathscr{L}$, we then define $\mathscr{\mathscr { F }}\left(B_{i}\right)=\left\{A \in \mathscr{F} \mid 2 A \cap l_{i} \neq \emptyset\right\}$. Let $\mathscr{H}=\left\{A \in \mathscr{F} \mid A \in \mathscr{F}\left(B_{i}\right)\right.$ for some $\left.B_{i} \in \mathscr{L}\right\}$.

(4) $A \in \mathscr{F}(B)$ implies $\rho(A) \geq\left(10^{3} /\left(10^{3}+3\right)\right) \rho(B)$.

(5) For $A \in \mathscr{H}$, let $A(\mathscr{L})=\{B \in \mathscr{L} \mid A \in \mathscr{F}(B)\}$. Then

$$
|A|^{-1} \sum_{B \in A(\mathscr{L})} \sharp \mathscr{F}(B)|B| \leq c\left(c_{1}\right) \log \left(\frac{R}{\rho(A)}\right) .
$$

In the following, for $A \subset M$, we denote $f_{A}=|A|^{-1} \int_{A} f d v_{M}, f_{A}^{\prime}=$ $\left(\int_{A} w d v_{M}\right)^{-1} \int_{A} f w d v_{M}$, and $|A|=\int_{A} d v_{M}$.

LEMMA 4.2. Let $M$ be a compact manifold with $C^{\infty}$ boundary $\partial M$. We assume that $M$ satisfies the following family of inequalities:

$$
\begin{aligned}
& \int_{B(x, r)}\left|f-f_{B(x, r)}\right|^{2} d v_{M} \leq c_{5} t^{2} r^{2} \int_{B(x, 8 r)}|\nabla f|^{2} d v_{M} \\
&+c_{5} t^{-v}|B(x, r)|^{-1}\left(\int_{B(x, r)}\left|f-f_{B(x, r)}\right| d v_{M}\right)^{2}, \\
& \forall B(x, 8 r) \subset \operatorname{Int} M, \quad \forall f \in C^{\infty}(\overline{B(x, 8 r)}), \forall t>0, \forall v \geq v_{0} .
\end{aligned}
$$

Moreover, we suppose that the pair $(M, w)$ satisfies properties $(D),\left(w_{1}\right)$, and $\left(w_{2}\right)$. Then, the Nash inequality (6) holds. 
Proof. Let $\mathscr{F}$ be a Whitney decomposition as in Lemma 4.1 and let $f \in C^{\infty}(\bar{M})$. By Lemma $4.1(1)$, for $B \in \mathscr{L}$, we can take $A_{1}, \ldots, A_{l} \in \mathscr{F}(B)$ satisfying

$$
A_{1}=B, \quad q \in 2 A_{l}, \quad 2 A_{i} \cap 2 A_{i+1} \neq \emptyset \quad(i=1,2, \ldots, l-1) .
$$

Let $l^{\prime}$ be the integer such that

$$
1 \leq l^{\prime} \leq l, \quad 4 A_{j} \nsubseteq M_{R / 2} \quad\left(j=1,2, \ldots, l^{\prime}-1\right), \quad 4 A_{l^{\prime}} \subset M_{R / 2} .
$$

Denote $f_{0}^{\prime}=f_{M_{R / 2}}^{\prime}=\int_{M_{R / 2}} f w d v_{M} / \int_{M_{R / 2}} w d v_{M}$.

Since $\int_{4 A_{i}}\left|f-f_{4 A_{i}}\right| \leq 2 \int_{4 A_{i}}\left|f-f_{4 A_{i}}^{\prime}\right|$, we have

$$
\int_{4 A_{i}}\left|f-f_{4 A_{i}}\right| w \leq c\left(c_{3}\right) \int_{4 A_{i}}\left|f-f_{4 A_{i}}^{\prime}\right| w .
$$

We note that for each $A_{i}$,

$$
x, y \in 32 A_{i} \Rightarrow w(x) \leq c_{3} w(y) .
$$

By (8), (9) and (10), we can see that

$$
\begin{aligned}
\int_{4 A_{i}}\left|f-f_{4 A_{i}}^{\prime}\right|^{2} w \leq & c\left(c_{3}, c_{5}\right) t^{2} \rho\left(A_{i}\right)^{2} \int_{32 A_{i}}|\nabla f|^{2} w \\
& +c\left(c_{3}, c_{5}\right) t^{-v}\left(\int_{4 A_{i}} w\right)^{-1}\left(\int_{4 A_{i}}\left|f-f_{4 A_{i}}^{\prime}\right| w\right)^{2}, \quad \forall t>0, \quad \forall v \geq v_{0} .
\end{aligned}
$$

For simplicity, we denote by $J_{A_{i}}(t)=J_{i}(t)$ the right hand side of the above inequality;

$$
\begin{aligned}
J_{A_{i}}(t)= & J_{i}(t) \\
= & c\left(c_{3}, c_{5}\right) t^{2} \rho\left(A_{i}\right)^{2} \int_{32 A_{i}}|\nabla f|^{2} w \\
& +c\left(c_{3}, c_{5}\right) t^{-v}\left(\int_{4 A_{i}} w\right)^{-1}\left(\int_{4 A_{i}}\left|f-f_{4 A_{i}}^{\prime}\right| w\right)^{2} .
\end{aligned}
$$

Then,

$$
\begin{aligned}
\left|f_{4 A_{i}}^{\prime}-f_{4 A_{i+1}}^{\prime}\right|^{2}\left(\int_{4 A_{i} \cap 4 A_{i+1}} w\right) & \leq 2 \int_{4 A_{i}}\left|f-f_{4 A_{i}}^{\prime}\right|^{2} w+2 \int_{4 A_{i+1}}\left|f-f_{4 A_{i+1}}^{\prime}\right|^{2} w \\
& \leq 2 J_{i}\left(t_{i}\right)+2 J_{i+1}\left(t_{i+1}\right), \quad\left(t_{i}>0, i=1,2, \ldots, l-1\right) .
\end{aligned}
$$

Since

$$
d\left(A_{i}, \partial M\right)=10^{3} \rho\left(A_{i}\right) \geq 10^{3} \frac{10^{3}}{10^{3}+3} \rho(B)=\frac{10^{3}}{10^{3}+3} d(B, \partial M),
$$

we see, by the assumption $\left(w_{1}\right)$,

$$
\frac{\int_{4 B} w}{\int_{4 A_{i}} w} \leq c\left(c_{1}, c_{3}\right) \frac{|B|}{\left|A_{i}\right|} .
$$


Therefore, since $4 A_{l^{\prime}} \subset M_{R / 2}$,

$$
\begin{aligned}
\left(\int_{4 B} w\right)\left|f_{4 A_{l^{\prime}}}^{\prime}-f_{0}^{\prime}\right|^{2} & =\frac{\int_{4 B} w}{\int_{4 A_{l^{\prime}}} w}\left(\int_{4 A_{l^{\prime}}}\left|f_{4 A_{l^{\prime}}}^{\prime}-f_{0}^{\prime}\right|^{2} w\right) \\
& \leq 2 \frac{\int_{4 B} w}{\int_{4 A_{l^{\prime}}} w}\left(\int_{4 A_{l^{\prime}}}\left|f-f_{4 A_{l^{\prime}}}^{\prime}\right|^{2} w+\int_{M_{R / 2}}\left|f-f_{0}^{\prime}\right|^{2} w\right) \\
& \leq c\left(c_{1}, c_{3}\right) \frac{|B|}{\left|A_{l^{\prime}}\right|}\left(J_{l^{\prime}}\left(t_{l^{\prime}}\right)+\int_{M_{R / 2}}\left|f-f_{0}^{\prime}\right|^{2} w\right) \quad\left(\forall t_{l^{\prime}}>0\right) .
\end{aligned}
$$

Hence,

$$
\begin{aligned}
\int_{4 B}\left|f-f_{0}^{\prime}\right|^{2} w \leq & 2 \int_{4 B}\left|f-f_{4 B}^{\prime}+\sum_{i=1}^{l^{\prime}-1}\left(f_{4 A_{i}}^{\prime}-f_{4 A_{i+1}}^{\prime}\right)\right|^{2} w+2\left(\int_{4 B} w\right)\left|f_{4 A_{l^{\prime}}}^{\prime}-f_{0}^{\prime}\right|^{2} \\
\leq & 2 l \int_{4 B}\left|f-f_{4 B}^{\prime}\right|^{2} w+2 l\left(\int_{4 B} w\right) \sum_{i=1}^{l^{\prime}-1}\left|f_{4 A_{i}}^{\prime}-f_{4 A_{i+1}}^{\prime}\right|^{2} \\
& +2\left(\int_{4 B} w\right)\left|f_{4 A_{l^{\prime}}}^{\prime}-f_{0}^{\prime}\right|^{2} \\
\leq & 2 l J_{1}\left(t_{1}\right)+4 l \sum_{i=1}^{l^{\prime}-1} \frac{\int_{4 B} w}{\int_{4 A_{i} \cap 4 A_{i+1}} w}\left\{J_{i}\left(t_{i}\right)+J_{i+1}\left(t_{i+1}\right)\right\} \\
& +c\left(c_{1}, c_{3}\right) \frac{|B|}{\left|A_{l^{\prime}}\right|}\left\{J_{l^{\prime}}\left(t_{l^{\prime}}\right)+\int_{M_{R / 2}}\left|f-f_{0}^{\prime}\right|^{2} w\right\}
\end{aligned}
$$

We note here that

$$
\frac{\int_{4 B} w}{\int_{4 A_{i} \cap 4 A_{i+1}} w} \leq c\left(c_{3}\right) \frac{|4 B|}{\left|4 A_{i} \cap 4 A_{i+1}\right|} \leq c\left(c_{1}, c_{3}\right) \min \left\{\frac{|B|}{\left|A_{i}\right|}, \frac{|B|}{\left|A_{i+1}\right|}\right\}
$$

and

$$
\left|A_{l^{\prime}}\right| \geq c\left(c_{1}, R / D\right)|M| .
$$

Indeed, the first inequality of (13) is due to (11).

When we take a point $y \in 2 A_{i} \cap 2 A_{i+1}$, we have

$$
B\left(y, 2 \min \left\{\rho\left(A_{i}\right), \rho\left(A_{i+1}\right)\right\}\right) \subset 4 A_{i} \cap 4 A_{i+1} .
$$

Since $10^{3} \rho\left(A_{i}\right)-10^{3} \rho\left(A_{i+1}\right)=d\left(A_{i}, \partial M\right)-d\left(A_{i+1}, \partial M\right) \leq \rho\left(A_{i}\right)+\rho\left(A_{i+1}\right), \quad$ we have also

$$
\left(10^{3}-1\right) \rho\left(A_{i}\right) \leq\left(10^{3}+1\right) \rho\left(A_{i+1}\right) .
$$

The second inequality of (13) follows from (15), (16) and the doubling property $(D)$. 
The inequality (14) is due to $(D)$ and the fact that $4 A_{l^{\prime}} \subset M_{R / 2}$. Indeed, by $(D)$, we have

$$
\left|A_{i}\right| \geq c_{1}^{-1}\left(\frac{\rho\left(A_{i}\right)}{D}\right)^{v_{0}}|M|, \quad v_{0}=\left(\log c_{1}\right) / \log 2 .
$$

And, since the fact that $4 A_{l^{\prime}} \subset M_{R / 2}$ implies $\rho\left(A_{l^{\prime}}\right) \geq R / 2\left(10^{3}-3\right)$, we have

$$
\left|A_{l^{\prime}}\right| \geq c_{1}^{-1}\left(\frac{R}{2\left(10^{3}-3\right) D}\right)^{v_{0}}|M|=c\left(c_{1}\right)\left(\frac{R}{D}\right)^{v_{0}}|M|,
$$

and (14) follows.

By (12), (13) and (14), we obtain

$$
\int_{4 B}\left|f-f_{0}^{\prime}\right|^{2} w \leq c\left(c_{1}, c_{3}\right) \sharp \mathscr{F}(B) \sum_{i=1}^{l^{\prime}} \frac{|B|}{\left|A_{i}\right|} J_{i}\left(t_{i}\right)+c\left(c_{1}, c_{3}\right)\left(\frac{D}{R}\right)^{v_{0}} \frac{|B|}{|M|} \int_{M_{R / 2}}\left|f-f_{0}^{\prime}\right|^{2} w .
$$

It is not hard to see that Lemma 4.1 (2) implies $A_{1}, A_{2}, \ldots, A_{l^{\prime}} \in \mathscr{L}$. Summing over all $B \in \mathscr{L}$, we get, by Lemma 4.1 (1), (3) and (5),

$$
\begin{aligned}
& \int_{\bigcup_{B \in \mathscr{L}} 4 B}\left|f-f_{0}^{\prime}\right|^{2} w \\
& \leq c\left(c_{1}, c_{3}\right) \sum_{B \in \mathscr{L}} \sharp \mathscr{F}(B) \sum_{A \in \mathscr{F}(B) \cap \mathscr{L}} \frac{|B|}{|A|} J_{A}\left(t_{A}\right)+c\left(c_{1}, c_{3}\right)\left(\frac{D}{R}\right)^{v_{0}} \int_{M_{R / 2}}\left|f-f_{0}^{\prime}\right|^{2} w \\
& \leq c\left(c_{1}, c_{3}\right) \sum_{A \in \mathscr{H} \cap \mathscr{L}}\left(|A|^{-1} \sum_{B \in A(\mathscr{L})} \sharp \mathscr{F}(B)|B|\right) J_{A}\left(t_{A}\right) \\
& +c\left(c_{1}, c_{3}\right)\left(\frac{D}{R}\right)^{v_{0}} \int_{M_{R / 2}}\left|f-f_{0}^{\prime}\right|^{2} w \\
& \leq c\left(c_{1}, c_{3}\right) \sum_{A \in \mathscr{H} \cap \mathscr{L}}\left(\log \left(\frac{R}{\rho(A)}\right)\right) J_{A}\left(t_{A}\right)+c\left(c_{1}, c_{3}\right)\left(\frac{D}{R}\right)^{v_{0}} \int_{M_{R / 2}}\left|f-f_{0}^{\prime}\right|^{2} w \\
& \leq c\left(c_{1}, c_{3}, c_{5}\right) \sum_{A \in \mathscr{H} \cap \mathscr{L}}\left\{\left(\frac{R}{\rho(A)}\right)^{\varepsilon_{1}} \varepsilon_{1}^{-1} \rho(A)^{2}\left(t_{A}\right)^{2} \int_{32 A}|\nabla f|^{2} w\right. \\
& \left.+\left(\frac{R}{\rho(A)}\right)^{\varepsilon_{2}} \varepsilon_{2}^{-1}\left(t_{A}\right)^{-v}\left(\int_{4 A} w\right)^{-1}\left(\int_{4 A}\left|f-f_{4 A}^{\prime}\right| w\right)^{2}\right\} \\
& +c\left(c_{1}, c_{3}\right)\left(\frac{D}{R}\right)^{v_{0}} \int_{M_{R / 2}}\left|f-f_{0}^{\prime}\right|^{2} w \quad\left(\forall t_{A}>0, \forall \varepsilon_{i}>0(i=1,2)\right) .
\end{aligned}
$$

For simplicity, we shall set

$$
I_{A}=\left(\frac{R}{\rho(A)}\right)^{\varepsilon_{1}} \varepsilon_{1}^{-1} \rho(A)^{2}\left(t_{A}\right)^{2} ; \quad I I_{A}=\left(\frac{R}{\rho(A)}\right)^{\varepsilon_{2}} \varepsilon_{2}^{-1}\left(t_{A}\right)^{-v}\left(\int_{4 A} w\right)^{-1 / 2}
$$


and put $t_{A}=\rho(A)^{-\theta}|A|^{-\delta} s$, where $s, \theta$ and $\delta>0$ are positive constants. Then, we have

$$
\begin{aligned}
I_{A} & =\varepsilon_{1}^{-1} R^{\varepsilon_{1}} \rho(A)^{2-\varepsilon_{1}-2 \theta}|A|^{-2 \delta} s^{2} \\
& \leq \varepsilon_{1}^{-1} R^{\varepsilon_{1}} \rho(A)^{2-\varepsilon_{1}-2 \theta-2 \delta v} c_{1}^{2 \delta}|M|^{-2 \delta} D^{2 \delta v} s^{2},
\end{aligned}
$$

where we have used the inequality (17) and the assumption $v \geq v_{0}$. We also have

$$
\begin{aligned}
I I_{A} & \leq c\left(c_{1}, c_{3}\right) \varepsilon_{2}^{-1} R^{\varepsilon_{2}} \rho(A)^{\theta v-\varepsilon_{2}}|A|^{\delta v-(1 / 2)} w\left(o_{A}\right)^{-1 / 2} s^{-v} \\
& \leq c\left(c_{1}, c_{3}, c_{6}\right) \varepsilon_{2}^{-1} R^{\varepsilon_{2}} \rho(A)^{\theta v-\varepsilon_{2}-\varphi}|A|^{\delta v-(1 / 2)} s^{-v},
\end{aligned}
$$

where we have used Lemma 4.1 (2) and the assumption $\left(w_{2}\right)$. For $v \geq v_{0}$ with $v>2 \varphi$, we set

Then we obtain

$$
\delta=\frac{1}{2 v} ; \quad \theta=\frac{1-\varepsilon_{1}}{2} ; \quad \varepsilon_{1}=\frac{v-2 \varphi}{2 v} ; \quad \varepsilon_{2}=\frac{v-2 \varphi}{4} .
$$

$$
\begin{aligned}
& \int_{\bigcup_{B \in \mathscr{L}} 4 B}\left|f-f_{0}^{\prime}\right|^{2} w \\
& \leq c\left(c_{1}, c_{3}, c_{5}, v\right)(v-2 \varphi)^{-1} R^{(v-2 \varphi) /(2 v)}|M|^{-1 / v} D s^{2} \int_{\bigcup_{A \in \mathscr{H} \cap \mathscr{L}} 32 A}|\nabla f|^{2} w \\
& \quad+c\left(c_{1}, c_{3}, c_{5}, c_{6}, v\right)(v-2 \varphi)^{-1} R^{(v-2 \varphi) / 4} s^{-v} \sum_{A \in \mathscr{H} \cap \mathscr{L}}\left(\int_{4 A} w\right)^{-1 / 2}\left(\int_{4 A}\left|f-f_{4 A}^{\prime}\right| w\right)^{2} \\
& \quad+c\left(c_{1}, c_{3}\right)\left(\frac{D}{R}\right)^{v_{0}} \int_{M_{R / 2}}\left|f-f_{0}^{\prime}\right|^{2} w .
\end{aligned}
$$

Since $\int_{4 A}\left|f-f_{4 A}^{\prime}\right| w \leq 2 \int_{4 A}|f-\alpha| w$ for all $\alpha \in \boldsymbol{R}$, and since $\int_{4 A}\left|f-f_{0}^{\prime}\right| w \leq$ $\left(\int_{4 A}\left|f-f_{0}^{\prime}\right|^{2} w\right)^{1 / 2}\left(\int_{4 A} w\right)^{1 / 2}$, we have

$$
\begin{aligned}
& \sum_{A \in \mathscr{H} \cap \mathscr{L}}\left(\int_{4 A} w\right)^{-1 / 2}\left(\int_{4 A}\left|f-f_{4 A}^{\prime}\right| w\right)^{2} \\
& \quad \leq 4 \sum_{A \in \mathscr{H} \cap \mathscr{L}}\left(\int_{4 A} w\right)^{-1 / 2}\left(\int_{4 A}\left|f-f_{0}^{\prime}\right| w\right)\left(\int_{4 A}\left|f-f_{M}^{\prime}\right| w\right) \\
& \quad \leq 4 \sum_{A \in \mathscr{H} \cap \mathscr{L}}\left(\int_{4 A}\left|f-f_{0}^{\prime}\right|^{2} w\right)^{1 / 2}\left(\int_{4 A}\left|f-f_{M}^{\prime}\right| w\right) \\
& \quad \leq c\left(c_{1}\right)\left(\int_{\bigcup_{A \in \mathscr{H} \cap \mathscr{L}} 4 A}\left|f-f_{0}^{\prime}\right|^{2} w\right)^{1 / 2} \int_{\bigcup_{A \in \mathscr{H} \cap \mathscr{L}} 4 A}\left|f-f_{M}^{\prime}\right| w \\
& \leq c\left(c_{1}\right)\left\{\varepsilon \int_{\bigcup_{A \in \mathscr{H} \cap \mathscr{L}} 4 A}\left|f-f_{0}^{\prime}\right|^{2} w+\varepsilon^{-1}\left(\int_{\bigcup_{A \in \mathscr{H} \cap \mathscr{L}} 4 A}\left|f-f_{M}^{\prime}\right| w\right)^{2}\right\}
\end{aligned}
$$


for all $\varepsilon>0$. Hence

$$
\begin{aligned}
& \int_{\bigcup_{B \in \mathscr{L}} 4 B}\left|f-f_{0}^{\prime}\right|^{2} w \\
& \leq c\left(c_{1}, c_{3}, c_{5}, v\right)(v-2 \varphi)^{-1} R^{(v-2 \varphi) /(2 v)}|M|^{-1 / v} D s^{2} \int_{\bigcup_{A \in \mathscr{H} \cap \mathscr{L}} 32 A}|\nabla f|^{2} w \\
& +c\left(c_{1}, c_{3}, c_{5}, c_{6}, v\right)(v-2 \varphi)^{-1} R^{(v-2 \varphi) / 4} s^{-v} \\
& \quad \times\left\{\varepsilon \int_{\bigcup_{A \in \mathscr{L}} 4 A}\left|f-f_{0}^{\prime}\right|^{2} w+\varepsilon^{-1}\left(\int_{\bigcup_{A \in \mathscr{L}} 4 A}\left|f-f_{M}^{\prime}\right| w\right)^{2}\right\} \\
& +c\left(c_{1}, c_{3}\right)\left(\frac{D}{R}\right)^{v_{0}} \int_{M_{R / 2}}\left|f-f_{0}^{\prime}\right|^{2} w .
\end{aligned}
$$

Therefore,

$$
\begin{aligned}
\{1- & \left.c\left(c_{1}, c_{3}, c_{5}, c_{6}, v, \varphi\right) R^{(v-2 \varphi) / 4} s^{-v} \varepsilon\right\} \int_{\bigcup_{A \in \mathscr{Q}} 4 A}\left|f-f_{0}^{\prime}\right|^{2} w \\
\leq & c\left(c_{1}, c_{3}, c_{5}, v, \varphi\right) R^{(v-2 \varphi) /(2 v)}|M|^{-1 / v} D s^{2} \int_{\bigcup_{A \in \mathscr{H} \cap \mathscr{L}} 32 A}|\nabla f|^{2} w \\
& +c\left(c_{1}, c_{3}, c_{5}, c_{6}, v, \varphi\right) R^{(v-2 \varphi) / 4} s^{-v} \varepsilon^{-1}\left(\int_{\bigcup_{A \in \mathscr{P}} 4 A}\left|f-f_{M}^{\prime}\right| w\right)^{2} \\
& +c\left(c_{1}, c_{3}\right)\left(\frac{D}{R}\right)^{v_{0}} \int_{M_{R / 2}}\left|f-f_{0}^{\prime}\right|^{2} w .
\end{aligned}
$$

In the above inequality, we shall choose $\varepsilon>0$ so that

$$
1 / 2=c\left(c_{1}, c_{3}, c_{5}, c_{6}, v, \varphi\right) R^{(v-2 \varphi) / 4} s^{-v} \varepsilon .
$$

Then, we have

$$
\begin{aligned}
\int_{M}\left|f-f_{0}^{\prime}\right|^{2} w \leq & \int_{\bigcup_{B \in \mathscr{L}} 4 B}\left|f-f_{0}^{\prime}\right|^{2} w+\int_{M_{R / 2}}\left|f-f_{0}^{\prime}\right|^{2} w \\
\leq & c\left(c_{1}, c_{3}, c_{5}, v, \varphi\right) R^{(v-2 \varphi) /(2 v)}|M|^{-1 / v} D s^{2} \int_{\bigcup_{A \in \mathscr{H} \cap \mathscr{L}} 32 A}|\nabla f|^{2} w \\
& +c\left(c_{1}, c_{3}, c_{5}, c_{6}, v, \varphi\right) R^{(v-2 \varphi) / 2} s^{-2 v}\left(\int_{\bigcup_{A \in \mathscr{L}} 4 A}\left|f-f_{M}^{\prime}\right| w\right)^{2} \\
& +\left(c\left(c_{1}, c_{3}\right)\left(\frac{D}{R}\right)^{v_{0}}+1\right) \int_{M_{R / 2}}\left|f-f_{0}^{\prime}\right|^{2} w
\end{aligned}
$$

for all $v$ satisfying $v \geq v_{0}$ and $v>2 \varphi$, and all $f \in C^{\infty}(\bar{M})$. 
In the following, we shall set $\sigma=2 v$ in the inequality (18), and prove the Nash inequality (6) with $v$ replaced by $\sigma$. Now, we shall consider the following Nash inequality on $M_{R / 2}$ :

$$
\begin{gathered}
\left(\int_{M_{R / 2}}\left|f-f_{M_{R / 2}}\right|^{2}\right)^{1+(2 / \sigma)} \leq N \int_{M_{R / 2}}|\nabla f|^{2} \cdot\left(\int_{M_{R / 2}}\left|f-f_{M_{R / 2}}\right|\right)^{4 / \sigma}, \\
N:=c_{N}\left(M_{R / 2}\right) \cdot\left|M_{R / 2}\right|^{-2 / \sigma} \cdot \operatorname{diam}\left(M_{R / 2}\right)^{2}, \quad \forall f \in C^{\infty}\left(\overline{M_{R / 2}}\right) .
\end{gathered}
$$

Since the assumption $\left(w_{1}\right)$ implies

$$
w(x) \leq c\left(c_{3}, D / R\right) w(y) \quad \forall x, \forall y \in M_{R / 2},
$$

it is not hard to see that

$$
\begin{aligned}
\int_{M_{R / 2}}\left|f-f_{M_{R / 2}}^{\prime}\right|^{2} w \leq & c\left(c_{3}, D / R, \sigma\right) N^{\sigma /(\sigma+2)}\left(\int_{M_{R / 2}}|\nabla f|^{2} w\right)^{\sigma /(\sigma+2)} \\
& \times\left(\int_{M_{R / 2}}\left|f-f_{M_{R / 2}}^{\prime}\right| w\right)^{4 /(\sigma+2)}\left(\sup _{M_{R / 2}} w\right)^{-2 /(\sigma+2)} .
\end{aligned}
$$

For $x_{0} \in \partial M_{R / 2},\left(w_{1}\right)$ and $\left(w_{2}\right)$ imply

$$
c_{6}(R / 2)^{2 \varphi} \leq w\left(x_{0}\right) \leq c\left(c_{3}, D / R\right) w(y) \quad\left(\forall y \in M_{R / 2}\right) .
$$

Hence, we have

$$
\left(\sup _{M_{R / 2}} w\right)^{-2 /(\sigma+2)} \leq c_{6}^{-2 /(\sigma+2)} c\left(c_{3}, D / R, \sigma\right) R^{-4 \varphi /(\sigma+2)} .
$$

We also have

$$
\int_{M_{R / 2}}\left|f-f_{M_{R / 2}}^{\prime}\right| w \leq 2 \int_{M_{R / 2}}\left|f-f_{M}^{\prime}\right| w .
$$

From (19), (20) and (21), we obtain

$$
\begin{gathered}
\int_{M_{R / 2}}\left|f-f_{M_{R / 2}}^{\prime}\right|^{2} w \leq c^{\prime}\left(\int_{M_{R / 2}}|\nabla f|^{2} w\right)^{\sigma /(\sigma+2)}\left(\int_{M_{R / 2}}\left|f-f_{M}^{\prime}\right| w\right)^{4 /(\sigma+2)}, \\
c^{\prime}=c\left(c_{3}, D / R, \sigma\right) c_{6}^{-2 /(\sigma+2)} R^{-4 \varphi /(\sigma+2)} N^{\sigma /(\sigma+2)} .
\end{gathered}
$$


Optimizing over $s>0$ in the inequality (18) with $v=\sigma / 2$, and using (22), we obtain

$$
\begin{aligned}
\int_{M}\left|f-f_{M_{R / 2}}^{\prime}\right|^{2} w \leq & c\left(c_{1}, c_{3}, c_{5}, c_{6}, \sigma, \varphi\right) R^{(\sigma-4 \varphi) /(\sigma+2)}|M|^{-2 /(\sigma+2)} D^{\sigma /(\sigma+2)} \\
& \times\left(\int_{\bigcup_{A \in \mathscr{H} \cap \mathscr{L}} 32 A}|\nabla f|^{2} w\right)^{\sigma /(\sigma+2)}\left(\int_{\bigcup_{A \in \mathscr{L}} 4 A}\left|f-f_{M}^{\prime}\right| w\right)^{4 /(\sigma+2)} \\
+ & c\left(c_{3}, D / R, \sigma\right) c_{6}^{-2 /(\sigma+2)} R^{-4 \varphi /(\sigma+2)} N^{\sigma /(\sigma+2)}\left(\int_{M_{R / 2}}|\nabla f|^{2} w\right)^{\sigma /(\sigma+2)} \\
& \times\left(\int_{M_{R / 2}}\left|f-f_{M}^{\prime}\right| w\right)^{4 /(\sigma+2)} \\
\leq & \mathscr{C}\left(\int_{M}|\nabla f|^{2} w\right)^{\sigma /(\sigma+2)}\left(\int_{M}\left|f-f_{M}^{\prime}\right| w\right)^{4 /(\sigma+2)}
\end{aligned}
$$

where we set

$$
\begin{aligned}
\mathscr{C}= & c\left(c_{1}, c_{3}, c_{5}, c_{6}, \sigma, \varphi\right) R^{(\sigma-4 \varphi) /(\sigma+2)}|M|^{-2 /(\sigma+2)} D^{\sigma /(\sigma+2)} \\
& +c\left(c_{3}, D / R, \sigma\right) c_{6}^{-2 /(\sigma+2)} R^{-4 \varphi /(\sigma+2)} N^{\sigma /(\sigma+2)} .
\end{aligned}
$$

Therefore, for all $\sigma>4 \varphi$ with $\sigma \geq 2 v_{0}$ and all $f \in C^{\infty}(\bar{M})$, we have

$$
\begin{aligned}
\left(\int_{M}\left|f-f_{M}^{\prime}\right|^{2} w\right)^{1+(2 / \sigma)} & \leq\left(\int_{M}\left|f-f_{M_{R / 2}}^{\prime}\right|^{2} w\right)^{1+(2 / \sigma)} \\
& \leq \mathscr{C}^{1+(2 / \sigma)}\left(\int_{M}|\nabla f|^{2} w\right)\left(\int_{M}\left|f-f_{M}^{\prime}\right| w\right)^{4 / \sigma} .
\end{aligned}
$$

Here, in general, since $(A+B)^{x} \leq 2^{x}\left(A^{x}+B^{x}\right)$ for positive constants $x, A$ and $B$, we have

$$
\begin{aligned}
\mathscr{C}^{1+(2 / \sigma)} \leq & c\left(c_{1}, c_{3}, c_{5}, c_{6}, \sigma, \varphi\right) R^{(\sigma-4 \varphi) / \sigma}|M|^{-2 / \sigma} D \\
& +c\left(c_{3}, D / R, \sigma\right) c_{6}^{-2 / \sigma} R^{-4 \varphi / \sigma} c_{N}\left(M_{R / 2}\right)\left|M_{R / 2}\right|^{-2 / \sigma} \operatorname{diam}\left(M_{R / 2}\right)^{2}
\end{aligned}
$$

Thus, we have the Nash inequality (6) with $v$ replaced by $\sigma$.

Theorem 4.1 follows from Lemma 3.1 and 4.2.

Remark 4.4. We note that the dumbbell-like example in R. Chen [4] shows that the interior rolling $R$-ball condition is necessary for the 'Nash constant' to be bounded from above. 


\section{An application}

In this section, as an application of Theorem 4.1, we shall derive the Sobolev inequality with respect to the measure $\left(u_{1}\right)^{2} d v_{M}$, where $u_{1}$ is the normalized first Dirichlet eigenfunction.

Let $M$ be a compact, connected Riemannian manifold with $C^{\infty}$ boundary $\partial M$. Denote by $v_{M}$ the Riemannian measure of $M$. We shall consider the following Dirichlet eigenvalue problem:

$$
\left\{\begin{array}{l}
\Delta u=-\lambda u \\
\left.u\right|_{\partial M} \equiv 0
\end{array}\right.
$$

Let $\left\{u_{i}\right\}_{i=1}^{\infty}$ be a complete orthonormal system of $L^{2}\left(M, v_{M}\right)$ consisting of Dirichlet eigenfunctions with $u_{i}$ having eigenvalue $-\lambda_{i}$. We shall take the sign of $u_{1}$ to be positive: $u_{1}>0$ on Int $M$. When we set $\phi_{i}=u_{i} / u_{1}$, a direct computation shows that $\phi_{i}$ satisfies the following equation:

$$
\Delta \phi_{i}+2 \nabla \log u_{1} \cdot \nabla \phi_{i}+\left(\lambda_{i}-\lambda_{1}\right) \phi_{i}=0 .
$$

Moreover, it is known that

$$
\phi_{i} \in C^{\infty}(\bar{M}), \quad \frac{\partial \phi_{i}}{\partial \vec{n}}=0,
$$

where $\vec{n}$ stands for the outward unit normal vector field on $\partial M$ (for this result, see [I. Singer-B. Wong-S. T. Yau-S. S. T. Yau [15]]). We shall define the closed form $\mathscr{E}_{u_{1}}$ on the Hilbert space $L^{2}\left(M,\left(u_{1}\right)^{2} v_{M}\right)$ in the following way:

$$
\begin{gathered}
\mathscr{E}_{u_{1}}(f, g)=\int_{M}\langle\nabla f, \nabla g\rangle\left(u_{1}\right)^{2} d v_{M}, \\
f, g \in \operatorname{Dom}\left(\mathscr{E}_{u_{1}}\right)=H^{1}\left(M,\left(u_{1}\right)^{2} v_{M}\right),
\end{gathered}
$$

where $H^{1}\left(M,\left(u_{1}\right)^{2} v_{M}\right)$ is the Hilbert space constructed by the completion of the space $C^{\infty}(\bar{M})$ with respect to the norm $\|f\|^{2}:=\mathscr{E}_{u_{1}}(f, f)+\int_{M} f^{2}\left(u_{1}\right)^{2} d v_{M}$. Let $A_{u_{1}}$ be the nonpositive self-adjoint operator on $L^{2}\left(M,\left(u_{1}\right)^{2} v_{M}\right)$ associated with $\mathscr{E}_{u_{1}}$. Then, we see that $\left\{\phi_{i}\right\}_{i=1}^{\infty}$ is a complete orthonormal system of $L^{2}\left(M,\left(u_{1}\right)^{2} v_{M}\right)$ consisting of eigenfunctions of $A_{u_{1}}$ with $\phi_{i}$ having eigenvalue $-\left(\lambda_{i}-\lambda_{1}\right)$. Setting $w=\left(u_{1}\right)^{2}$ and applying Theorem 1.3 in [13] and Theorem 3.7 in [J. Wang [17]], we have the following

Proposition 5.1. We assume that Int $M=M-\partial M$ is a relatively compact domain in a complete Riemannian manifold $L$ with its Ricci curvature $\mathrm{Ric}_{L}$ bounded from below by $-K$ for some constant $K \geq 0$. Moreover, we suppose that $M$ satisfies the interior rolling $R$-ball condition $(R)$ and that the second fundamental form $\Pi_{\partial M}$ of $\partial M$ with respect to the outward unit normal $\vec{n}$ is bounded from above by $H$ for some constant $\left.H \geq a^{\prime}\right\rangle 0$, that is, $\Pi_{\partial M}(X, X):=-\left\langle\nabla_{X}(\partial / \partial \vec{n}), X\right\rangle \leq$ 
$H|X|^{2}$, where $-\left(a^{\prime}\right)^{2}$ is a lower bound of the sectional curvature on $M-M_{R / 2}$. Then, we have, for any $v$ satisfying $v \geq 2 v_{0}$ and $v>4$, there exists a constant $c(v, K, H, R, D, \operatorname{dim} M, \operatorname{Vol}(M))$ such that the following Sobolev inequality holds:

$$
\inf _{\alpha \in \boldsymbol{R}}\left(\int_{M}|f-\alpha|^{2 v /(v-2)}\left(u_{1}\right)^{2} d v_{M}\right)^{(v-2) / v} \leq c^{\prime} \int_{M}|\nabla f|^{2}\left(u_{1}\right)^{2} d v_{M}, \quad f \in C^{\infty}(\bar{M}),
$$

where $D$ stands for the intrinsic diameter of $M, v_{0}=v_{0}(K, R)=\left(\log c_{1}\right) / \log 2$ and $c_{1}$ is a volume doubling constant on $M-M_{R}$ as before.

Proof. The property $(P)$ is satisfied by the fact $\operatorname{Ric}_{M} \geq-K$ (see example 1 in [14]). In the similar way to the one in [13], we can prove that for $w=\left(u_{1}\right)^{2}, M$ satisfies the conditions $\left(w_{1}\right)$ and $\left(w_{2}\right)$ with $\varphi=1, c_{3}=$ $c(K, H, R, D, \operatorname{dim} M, \operatorname{Vol}(M)) \quad$ and $\quad c_{6}=c(K, H, R, D, \operatorname{dim} M, \operatorname{Vol}(M))$. We note that, by the comparison theorem and by the argument about the interval of the existance of solution, we can see that the second fundamental form of $M_{R / 2}$ satisfies $-c(R, H, \operatorname{dim} M) \leq \Pi_{\partial M_{R / 2}} \leq \max \left\{H, a^{\prime}\right\}=H$. Therefore, we also have

$$
c_{N}\left(M_{R / 2}\right)\left|M_{R / 2}\right|^{-2 / v} \operatorname{diam}\left(M_{R / 2}\right) \leq c\left(v, \operatorname{dim} M, H, R, K,\left|M_{R / 2}\right|, \operatorname{diam}\left(M_{R / 2}\right)\right),
$$

by Theorem 3.7 in [17] and by [4], where $\operatorname{diam}\left(M_{R / 2}\right)$ is the intrinsic diameter of $M_{R / 2}$. Hence, Proposition 5.1 easily follows from Theorem 4.1.

Corollary 2.1 immediately implies the following

COROllary 5.1. The inequality (23) implies the following estimate:

$$
\alpha^{2 / v}(c v)^{-1} \leq \lambda_{2}-\lambda_{1} .
$$

Moreover, (23) implies the decay of eigenfunctions:

$$
u_{i}(x) \leq \alpha\left(v c\left(\lambda_{i}-\lambda_{1}\right)\right)^{v / 4} u_{1}(x) \quad(i=2,3, \ldots),
$$

where $\alpha$ is some numerical constant.

Remark 5.1. Actually, the optimal value of the constant $v$ in Proposition 5.1 is $\operatorname{dim} M+2$. This fact and other results will be proved in H. Kumura [12].

\section{REFERENCES}

[1] K. Akutagawa, Yamabe metrics of positive scalar curvature and conformally flat manifolds, Differential Geom. Appl., 4 (1994), 239-258.

[2] E. Carlen, S. Kusuoka ANd D. Stroock, Upper bounds for symmetric Markov transition functions, Ann. Inst. H. Poincaré Probab. Statist., 23 (1987), 245-287.

[3] I. Chavel, Eigenvalues in Riemannian Geometry, Academic Press, New York, 1984.

[4] R. CHEN, Neumann eigenvalue estimate on a compact Riemannian manifold, Proc. Amer. Math. Soc., 108 (1990), 961-970.

[5] R. Chen and P. Li, On Poincaré type inequalities, Trans. Amer. Math. Soc., 349 (1997), $1561-1585$. 
[6] S. Y. Cheng ANd K. Oden, Isoperimetric inequalities and the gap between the first and second eigenvalues of an Euclidean domain, J. Geom. Anal., 7 (1997), 217-239.

[ 7 ] E. B. Fabes AND D. W. Strook, A new proof of Moser's parabolic Harnack inequality using the old idea of Nash, Arch. Rat. Mech. Anal., 96 (1986), 327-338.

[8] A. A. GRIGOR'YAN, The heat equation on noncompact Riemannian manifolds, Math. USSRSb., 72 (1) (1992), 47-77.

[ 9 ] P. Hajlasz and P. Koskela, Sobolev met Poincaré, Mem. Amer. Math. Soc., 145 (2000), no. 688.

[10] D. Jerison, The Poincaré inequality for vector fields satisfying Hörmander's condition, Duke Math. J., 53 (1986), 503-523.

[11] A. Kasue and H. Kumura, Spectral convergence of Riemannian manifolds, II, Tôhoku Math. J., 48 (1996), 71-120.

[12] H. Kumura, On the intrinsic ultracontractivity for compact manifolds with boundary, preprint.

[13] K. Oden, C. J. Sung And J. Wang, Spectral gap estimates on compact manifolds, Trans. Amer. Math. Soc., 351 (1999), 3533-3548.

[14] L. Saloff-Coste, A note on Poincaré, Sobolev and Harnach inequality, Internat. Math. Res. Notices, 2 (1992), 27-38.

[15] I. M. Singer, B. Wong, S. T. Yau and S. S. T. Yau, An estimate of the gap of the first two eigenvalues in the Schrödinger operator, Ann. Scuola Norm. Sup. Pisa Cl. Sci. (4), 12 (1985), 319-333.

[16] N. Varopoulos, Hardy-Littlewood theory for semigroups, J. Funct. Anal., 63 (1985), 240 260.

[17] J. Wang, Global heat kernel estimates, Pacific J. Math., 178 (1997), 377-398.

Department of Mathematics

SHIZUOKA UNIVERSITY

OHYA, SHIZUOKa 422-8529

JAPAN

e-mail: smhkumu@ipc.shizuoka.ac.jp 